\title{
The role of lactose in weanling pig nutrition: a literature and meta-analysis review
}

\author{
Jinbiao Zhao ${ }^{1,2}$, Zeyu Zhang ${ }^{1,2}$, Shuai Zhang ${ }^{1}$, Greg Page ${ }^{2}$ and Neil W. Jaworski ${ }^{2^{*}}$ (D)
}

\begin{abstract}
Lactose plays a crucial role in the growth performance of pigs at weaning because it is a palatable and easily digestible energy source that eases the transition from milk to solid feed. However, the digestibility of lactose declines after weaning due to a reduction in endogenous lactase activity in piglets. As a result, some lactose may be fermented in the gastrointestinal tract of pigs. Fermentation of lactose by intestinal microbiota yields lactic acid and volatile fatty acids, which may positively regulate the intestinal environment and microbiome, resulting in improved gastrointestinal health of weanling pigs. We hypothesize that the prebiotic effect of lactose may play a larger role in weanling pig nutrition as the global feed industry strives to reduce antibiotic usage and pharmacological levels of zinc oxide and supra-nutritional levels of copper. Evidence presented in this review indicates that high dietary lactose improves growth performance of piglets, as well as the growth of beneficial bacteria, particularly Lactobacillus, with the positive effects being more pronounced in the first 2 weeks after weaning. However, the risk of post-weaning diarrhea may increase as pigs get older due to reduced lactase activity, high dietary lactose concentrations, and larger feed intakes, all of which may lead to excessive lactose fermentation in the intestine of the pig. Therefore, dietary lactose levels exert different effects on growth performance and gastrointestinal physiological functions in different feeding phases of weanling pigs. However, no formal recommendation of lactose for weanling pigs has been reported. A meta-analysis approach was used to determine that diets fed to swine should include 20\%, 15\%, and 0 lactose from d 0-7, d 7-14, and d 14-35 post-weaning, respectively. However, sustainable swine production demands that economics must also be taken into account as lactose and lactose containing ingredients are expensive. Therefore, alternatives to lactose, so called "lactose equivalents" have also been studied in an effort to decrease feed cost while maintaining piglet performance with lower dietary lactose inclusions. In summary, the present review investigated dose-response effects of dietary lactose supplementation to exert positive responses and begin to elucidate its mechanisms of action in postweaning pig diets. The results may help to replace some or all lactose in the diet of weanling pigs, while improving production economics given the high cost of lactose and availability in some swine production markets.
\end{abstract}

Keywords: Growth performance, Gut health, Gut microbiota, Lactose, Lactose equivalents, Weanling pig

\footnotetext{
* Correspondence: neil.jaworski@trouwnutrition.com

${ }^{2}$ Trouw Nutrition Innovation, Stationsstraat 77, 3800AG Amersfoort, Netherlands

Full list of author information is available at the end of the article
}

(c) The Author(s). 2021 Open Access This article is licensed under a Creative Commons Attribution 4.0 International License, which permits use, sharing, adaptation, distribution and reproduction in any medium or format, as long as you give appropriate credit to the original author(s) and the source, provide a link to the Creative Commons licence, and indicate if changes were made. The images or other third party material in this article are included in the article's Creative Commons licence, unless indicated otherwise in a credit line to the material. If material is not included in the article's Creative Commons licence and your intended use is not permitted by statutory regulation or exceeds the permitted use, you will need to obtain permission directly from the copyright holder. To view a copy of this licence, visit http://creativecommons.org/licenses/by/4.0/. The Creative Commons Public Domain Dedication waiver (http://creativecommons.org/publicdomain/zero/1.0/) applies to the data made available in this article, unless otherwise stated in a credit line to the data. 


\section{Introduction}

Lactose is a disaccharide present in milk and is the main carbohydrate source for infant mammals. Lactose is digested by lactase into glucose and galactose in the small intestine providing readily absorbable energy for young mammals [1]. A portion of dietary lactose is fermented by bacteria, like Lactobacillus, in the stomach, producing lactic acid and minimal quantities of acetate [2], which maintain gastric acidity in suckling piglets [3]. Endogenous lactase activity in pigs sharply declines at weaning [4]. Piglets are weaned, on average in the global swine industry, 3 to 4 weeks after birth, which results in a decreased capacity to digest high levels of lactose [4]. Recent research in humans suggests the decrease in lactase activity to be independent of the amount of lactose and the duration of lactose intake in the diet [5]. Therefore, a certain amount of lactose may reach the large intestine of the pig and be a substrate for microbial fermentation to produce lactic acid and volatile fatty acids (VFA), which have been reported to be beneficial to gut health and host metabolism [6, 7].

The prebiotic effect of lactose may now play a larger role in weanling pig nutrition as the global feed industry strives to reduce antibiotic usage, pharmacological levels of zinc oxide $(\mathrm{ZnO})$, and supra-nutritional levels of $\mathrm{Cu}$. Recent studies, especially in the human infant and lactose intolerant realms, have focused on exploring the prebiotic effect of lactose. Lactose has been described as a prebiotic that exerts health benefits through selective stimulation of intestinal bifidobacteria and lactobacilli in adults with impaired lactose digestion and without a history of gastrointestinal disease $[8,9]$. Some studies reported that the prebiotic health benefits of lactose were not only associated with intestinal microbial composition, but also related to many different microbial metabolites, one of which is butyrate, which is an important energy source for the colonic mucosa and has antiinflammatory effects amongst several other health benefits $[10,11]$. Moreover, acid production in the gastrointestinal tract has been shown to modulate the composition of gut microbiota by decreasing intestinal $\mathrm{pH}$, thereby reducing growth and proliferation of some intestinal pathogens [12]. However, an explicit requirement for dietary lactose in weanling piglets is not available [13]. Suckling and weanling pigs most probably do have a specific requirement for lactose as they consume a milk-based diet from the sow and transition onto a lower lactose creep feed, to an even lower lactose concentrated solid feed after weaning. On the other hand, excessive intake and over-fermentation of lactose may lead to intolerance symptoms in humans resulting in diarrhea and this may also occur in pigs as their gastrointestinal system is very similar to humans [5]. Therefore, dietary lactose supplementation in weanling pig nutrition may be a balancing act between providing an easily digestible energy source and an easily fermentable prebiotic source. This review investigated mechanisms of action of lactose when included in post-weaning swine diets with the aim to disclose nutritional and prebiotic effects and the optimal concentration of lactose in weanling pig diets.

\section{Definition of lactose}

Lactose is a disaccharide consisting of $\alpha-D$-glucose and $\beta$ - $D$-galactose, which is chemically formed as $O-\beta-D$ galactopyranosyl-(1-4)- $\beta$-glucose [14]. Carbon 1 of the glucose moiety is anomeric because it carries a hydroxyl group which is free to lie above or below the plane of the ring. This hydroxyl group is responsible for the existence of $\alpha$ - and $\beta$-lactose forms, which can be interconverted in aqueous solution under different processing temperatures. $\alpha$-lactose crystallizes from super-saturated solutions at temperatures below $93.5^{\circ} \mathrm{C}$ to produce a variety of crystal shapes, but above $93.5^{\circ} \mathrm{C}$, $\beta$-lactose is transformed as an uneven-sided diamond. $\beta$ lactose is more easily dissolved in water and sweeter compared to $\alpha$-lactose. Therefore, $\beta$-lactose may improve pig feed intake to a greater extent compared with $\alpha$-lactose, but no studies have tested this hypothesis. $\alpha$ lactose is primarily used in the pharmaceutical industry as a carrier for pill manufacturing because of its stability and good storage properties [15]. However, anomeric composition of the wide range of commercially available lactose sources has not been investigated and the development of rapid methods for distinguishing the anomeric composition of lactose products may be warranted.

\section{Lactose in sow milk}

The disaccharide lactose occurs almost exclusively in the milk of mammals. Glucose is the sole precursor of lactose in animals and the synthesis of lactose from glucose requires a number of enzyme-mediated steps (Fig. 1). Importantly, glucose must be derived from nutrient digestion due to a lack of glucose-6-phosphatase in the sow, then be readily absorbed from the small intestine into the bloodstream, and then flow into mammary arterial blood [16].

Lactose concentration in milk differs among animal species. Human milk contains $60-70 \mathrm{~g} / \mathrm{L}$ lactose in addition to $12-15 \mathrm{~g} / \mathrm{L}$ milk oligosaccharides. While the milk of goat, sheep, and cow, all contain between 40 and $50 \mathrm{~g} / \mathrm{L}$ lactose and only a small fraction of milk oligosaccharides [17]. More similar to human milk, sow colostrum and milk contain $40 \mathrm{~g} / \mathrm{L}$ and $60 \mathrm{~g} / \mathrm{L}$ of lactose, respectively $[18,19]$. The milk oligosaccharide content of sow milk is as high as $23 \mathrm{~g} / \mathrm{L}$ in colostrum and rapidly decreases during lactation to values as low as 5 to $10 \mathrm{~g} / \mathrm{L}$ in late lactation. The primary porcine milk 


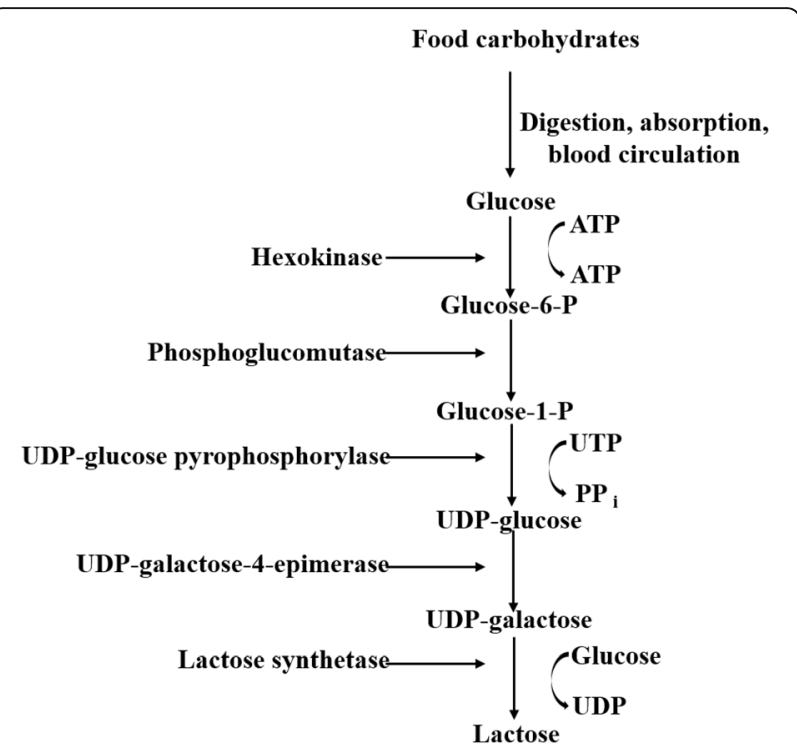

Fig. 1 The biosynthesis of lactose in the mammary gland of sows (adapted from Blackburn et al. [16])

oligosaccharides (MOs) are fucosylated-MOs, sialylatedMOs, and neutral-MOs and these act as prebiotics for suckling pigs [20].

Milk and lactose yield in sows were reported to be associated with type of diet, lactation environment, suckling frequency of piglets, and health status [21]. Temperatures on either side of the thermoneutral zone, in addition to high relative humidity, have been shown to decrease milk lactose yield in lactating sows [22]. Zhao et al. [23] reported that the increased proportion of valine to lysine from $0.72 \%$ up to $1.01 \%$ in the diet of sows from d 29 prepartum to $\mathrm{d} 21$ postpartum increased lactose concentration of sow colostrum in a hot and humid environment. Dunshea et al. [24] infused a combination of insulin $(11 \mathrm{mU} / \mathrm{kg}$ per hour) and dextrose (50\%) into the anterior vena cava of sows using silastic catheters during mid (d 5-10) and late (d 17-22) lactation, and this resulted in increased concentration of lactose in milk. Crude protein concentrations of $16 \%$ or $23 \%$ supplied by varying quantities of fish meal, blood meal and branched-chain amino acids in diets fed to sows throughout lactation had no effect on lactose concentration in milk [24]. Dourmad et al. [25] also observed that lactating sows fed diets containing different concentrations of crude protein $(15.5 \%$ or $17 \%)$ or lysine $(0.66 \%, 0.77 \%$, or $0.87 \%)$ had no effect on sow milk lactose content. Other reports have also indicated that sows fed diets containing higher levels of branched-chain amino acids, tryptophan, or lysine had no effect on sow milk lactose concentration [18, 26]. Furthermore, Yang et al. [27] determined that sows fed 13.7, 13.9, or 14.2 MJ metabolizable energy (ME) per $\mathrm{kg}$ during late gestation and lactation did not affect lactose content in sow milk. The supplementation of $0.8 \%$ or $1.2 \%$ potassium diformate to diets fed from mating until the next mating, or $0.2 \%$ betaine from $5 \mathrm{~d}$ before the expected date of farrowing until the end of lactation had no effect on the lactose concentration of sow milk or colostrum [28, 29]. Overall, literature evidences suggest that sow milk and colostrum lactose concentrations are difficult to change through dietary macronutrient density or addition of different feed additives in sow diets.

\section{Digestion and absorption of lactose in suckling and weanling pigs}

Piglets are born with high intestinal lactase activity and are generally able to fully digest dietary lactose. Lactase belongs to a group of intestinal disaccharidases located on the brush border of the small intestine. Normally, the activity of lactase is highest in the proximal part of the jejunum and progressively declines towards the ileum [30]. Lactase hydrolyses lactose into the monosaccharide sugars glucose and galactose, which are then available for absorption [1]. Lactase gene expression is initiated before the birth of piglets, remains high during nursing, and then sharply declines after weaning [30]. Feeding neonatal piglets for $6 \mathrm{~h}$ with milk replacer, instead of porcine colostrum, was shown to significantly decrease total lactase activity by $25 \%$ [31]. De Vos et al. [32] reported feeding colostrum and milk rapidly induced an increase in lactase activity ( $7 \mathrm{U} / \mathrm{g}$ of tissue) compared with those fed a formulated $\operatorname{diet}(3 \mathrm{U} / \mathrm{g}$ of tissue), especially during the first week post-farrow. However, a significant decline in lactase activity in the small intestine of weanling pigs was observed between 3 and 5 weeks of age despite the presence of considerable quantities (19\%) of dietary lactose [4], which suggests that mucosal lactase activity is independent of the amount of lactose and the duration of lactose intake in the diet [5]. Weaning, therefore, appears to be the major cause of reduced lactase activity and this is independent of lactose concentration in the diet and duration of feeding lactose containing diets. This implies that some lactose may escape digestion due to reduced lactase activity after weaning and this may result in excessive fermentation of lactose by gut microbiota and exacerbate enteric diseases resulting in post-weaning diarrhea. Indeed, weanling pigs fed a diet containing $29.5 \%$ lactose from d 14-21 postwean resulted in increased diarrhea compared with pigs fed a diet containing $17.5 \%$ lactose due to reduced lactase activity and excessive lactose fermentation in the large intestine [33]. It is hypothesized that diarrhea induced by a high concentration of lactose in diets may be a result of poor microbial adaptation to a large flow of easily and, most probably, rapidly fermentable lactose.

In newborn piglets, acid secretion is low in the stomach. The principal source of stomach acidity is from 
bacterial fermentation of lactose into lactic acid as well as acetate, which activates endogenous enzymes and gut microbiota and improves gut health of suckling pigs [34]. Some lactose will escape bacterial fermentation in the stomach and flow into the small intestine to be enzymatically hydrolyzed by endogenous lactase to be used as an energy source $[35,36]$. In addition to endogenous lactase activity, many bacteria in the gastrointestinal tract of pigs express $\beta$-galactosidase activity that allow microbes to utilize lactose and produce lactic acid and VFA through fermentation [37]. This is most probably beneficial in the foregut, where bifidobacteria and Lactobacillus dominate, especially in a healthy, nonchallenged environment. However, this could be detrimental in a disease challenged gut as E. coli and streptococci also possess $\beta$-galactosidase.

The adult pig, on the other hand, has limited lactase activity in the small intestine and, therefore, lactose may be a significant substrate for microbial fermentation in the small and large intestine. Pierce et al. [38] reported that butyric acid concentration linearly increased and a ratio of acetic acid to propionic acid linearly decreased in the feces of growing-finishing pigs fed diets containing 0 up to $12 \%$ lactose. These results indicate that a certain amount of lactose entered the hindgut and was fermented causing shifts in microbial metabolite production. Williams et al. [39] reported that enteric health was improved when the fermentation of lactose occurs in the large intestine. Lactose can be fermented to generate lactic acid and VFA by gut bacteria in the hindgut of pigs [33], which improve intestinal health by modifying gut microbiota and induce the expression of porcine host defense peptides, such as pBD2, pBD3 and pEP2C $[6,7]$. Previous studies have shown improved insulin sensitivity through VFA-mediated glucose homeostasis by activating G protein-coupled receptors 41 and 43 and stimulating enteroendocrine L-cells to produce glucagon-like peptide 1 and peptide YY [40, 41]. Overall, lactic acid and VFA produced by intestinal microbiota to ferment lactose play a crucial role in improving intestinal health of piglets [42].

\section{Effect of lactose level on growth performance and diarrhea}

Dietary lactose supplementation was shown to improve the growth performance of post-weaned pigs [43]. The positive effect of dietary lactose on weanling piglets was mainly attributed to its sweetness resulting in improved diet palatability observed through increased average daily feed intake (ADFI). In addition, Partridge and Gill [44] attributed some of the improved growth performance to reduced stomach $\mathrm{pH}$ post-weaning through production of lactic acid and VFA from lactose fermentation. Furthermore, a lower $\mathrm{pH}$ in the stomach inhibits pathogen growth and improves protein digestion [45]. The inclusion of $25 \%$ lactose improved feed intake and weight gain of piglets weaned at 3 to 4 weeks compared with piglets fed a diet containing only $15 \%$ lactose from $\mathrm{d} 0$ 7 after weaning [46-48]. A linear increase in average daily gain (ADG) and a quadratic increase in feed efficiency (G:F) were observed during the second week after weaning in pigs weaned at 24 days old with an average initial body weight (BW) of $7.1 \mathrm{~kg}$ and fed diets containing $6.5 \%, 17.0 \%$, or $28.0 \%$ lactose [49]. In a follow-up study, pigs with an average initial BW of $6 \mathrm{~kg}$ (weaned at 24 days old) and fed a diet containing $29.5 \%$ lactose had greater ADFI and ADG than those fed a diet with 17.5\% lactose during the first week after weaning [33]. On the other hand, piglets (weaned at 23 days old and an average initial BW of $6.5 \mathrm{~kg}$ ) fed a diet containing 35.5\% lactose had lower ADG compared with pigs fed a diet with $22.5 \%$ lactose in the first 2 weeks post-wean [50]. Pierce et al. [33] reported that piglets fed a diet with $29.5 \%$ lactose had lower diarrhea incidence in the first week after weaning, but showed higher diarrhea incidence in the second and third weeks compared with pigs fed a $17.5 \%$ lactose diet. These results indicate that a level of $29.5 \%$ lactose in the diet may have exceeded the lactase activity of pigs between 2 and 3 weeks post-weaning, resulting in excessive fermentation of lactose and incidence of diarrhea. Bertol et al. [51] found lactose levels up to $21 \%$ in diets for piglets weaned at $21 \mathrm{~d}$ of age linearly increased the ADFI and ADG of pigs in the first 14 days postweaning. Mahan et al. [52] reported piglets with an average initial BW of $6.3 \mathrm{~kg}$, weaned at $19 \mathrm{~d}$ of age, and fed diets containing $10 \%$ to $35 \%$ lactose in the first 2 weeks post-weaning, showed a linear increase in ADG and improved G:F in the first week. Increasing the dietary lactose levels $(0,10 \%$, and $15 \%)$ in the first 2 weeks after weaning enhanced growth performance of piglets (mean initial BW of $6.4 \mathrm{~kg}$ and average age of $18 \mathrm{~d}$ ) fed using a liquid feeding system, but this advantage was not maintained after 2 weeks post-wean [53]. Similarly, weanling pigs with initial BW of $6 \mathrm{~kg}$ (weaned at 20 days old) that received a diet with $20 \%$ lactose in the first 2 weeks had greater growth performance, although no positive effects were observed after 3 weeks post-wean when pigs were fed a diet containing 15\% lactose [54]. Taken together, these results indicate that the positive effect of lactose on performance of weanling piglets mainly exist in the first 2 weeks after weaning. This is in general alignment with the observed declines in endogenous lactase activity noted earlier. Interestingly, piglets fed high lactose from $4 \mathrm{~d}$ of age to weaning showed greater growth performance in the nursery period, which persisted throughout the finisher and ended in greater slaughter weights, as well as hot and cold carcass weights, compared with a low lactose diet [55]. Piglets weaned at 21 or $28 \mathrm{~d}$ of age, 
and fed a diet with $25 \%$ lactose from d $0-7$ post-wean, had a positive response on subsequent performance in the whole nursery period in comparison with piglets fed 15\% lactose [48]. These results indicate that high dietary lactose in the first 2 weeks post-weaning may be beneficial to performance of pigs in subsequent growth phases.

On the other hand, there have been studies which reported no or a negative response to increased lactose levels in diets fed to weanling pigs. Pollmann et al. [56] found adding $10 \%$ lactose to the diet did not improve growth performance of piglets (initial BW of $6 \mathrm{~kg}$ ) assessed weekly until $28 \mathrm{~d}$ post weaning. Furthermore, no growth performance response was observed when pigs (initial BW of $7.05 \mathrm{~kg}$ and weaned at 24 days old) were fed diets containing $10 \%$ or $20 \%$ lactose in the first 2 weeks post-wean [57]. Lactose included in diets at 0 , $4 \%, 8 \%$ and $12 \%$ did not affect ADG, ADFI, and G:F of weanling pigs (initial BW of $6.1 \mathrm{~kg}$ and weaned at $21 \mathrm{~d}$ of age) in the first 2 weeks after weaning [58].

The inconsistent observations reported in growth performance of pigs fed diets with higher lactose concentrations may be associated with feeding environment, dietary ingredient and nutrient composition, and weaning age. But, most importantly, weaning stress impacts postweaning pig performance to a great extent and is the most probable cause for variation in pig response to different levels of dietary lactose. Mahan et al. [52] recommended that diets fed to pigs (weaned at 19 days old and average initial BW of $6.4 \mathrm{~kg}$ ) from d $0-7, \mathrm{~d} 7-21$, and d 21-35 post-wean should contain $25-30 \%, 15-20 \%$, and $10-15 \%$ lactose, respectively. Cromwell et al. [59] recommended that dietary lactose should be $20 \%$ in diets fed from $\mathrm{d} 0-7$ post-wean, $15 \%$ in diets fed from $\mathrm{d} 7-14$ post-wean, and $7.5 \%$ in diets fed from d $14-30$ post-wean.

\section{Model to predict performance responses of weanling pigs fed different concentrations of dietary lactose using a meta-regression technique} Growth performance (ADFI, ADG, and G:F) of weanling pigs fed diets containing different levels of lactose were modelled using a meta-regression technique in the present review. First, a database was constructed from published literature which reported growth performance of weanling pigs fed different levels of dietary lactose. Studies were from published articles in scientific journals indexed in public data search generators (PubMed, Web of Science and Science Direct). The literature search, screening, and data extraction processes are shown in Fig. 2. The inclusion criteria for studies were as follows: 1) peerreviewed and published in the English language from 1990 to 2019; 2) lactose study on weanling pigs; 3) dietary lactose levels as the sole source of variation. A total of 14 published studies were used to create response models that predicted the performance of weanling pigs fed varying levels of dietary lactose (Table 1).

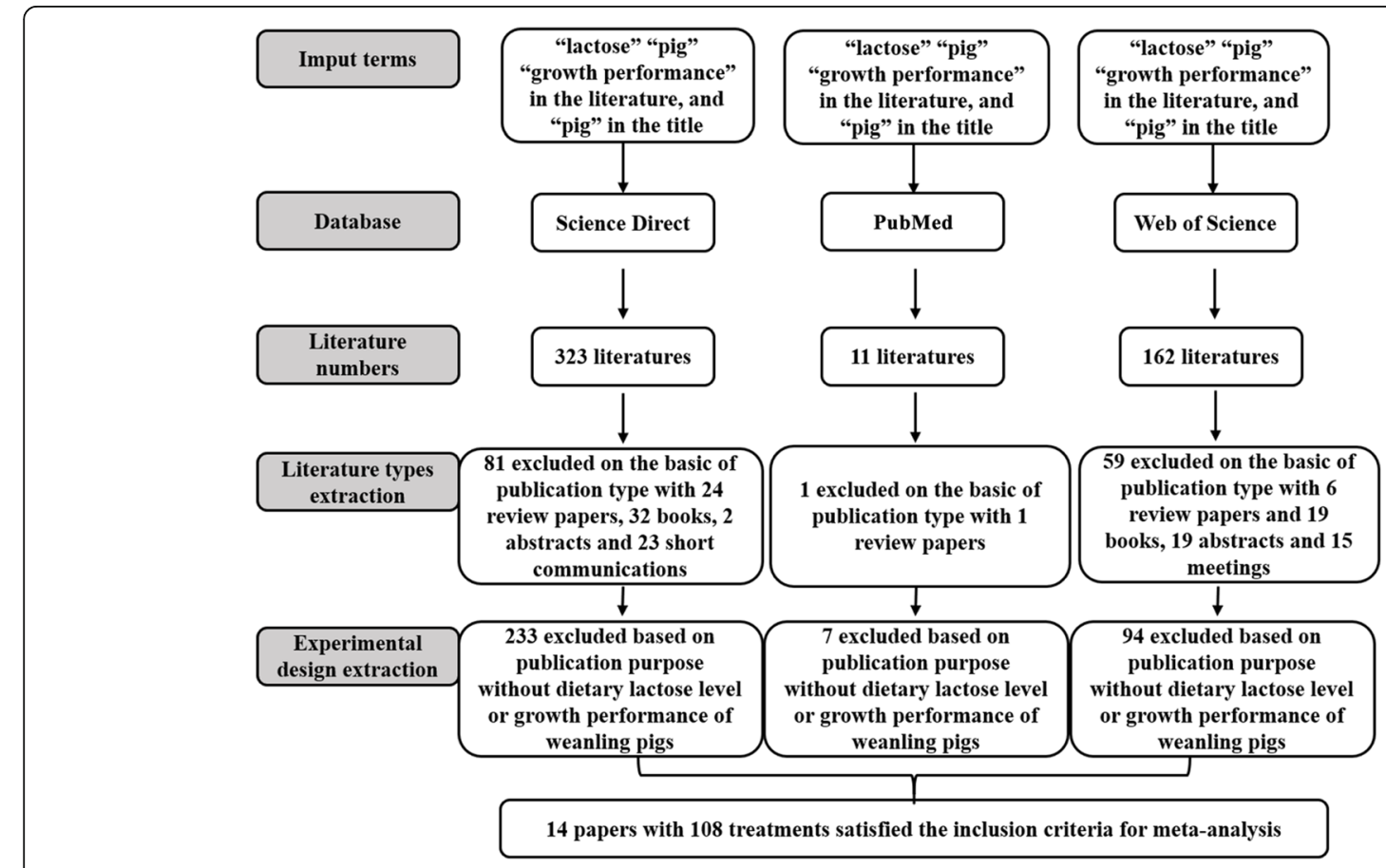

Fig. 2 Flow chart of the literature search, screening and data extraction procedures 
Table 1 Summary of studies evaluating the effect of dietary lactose concentration on growth performance of weanling pigs used in the meta-analysis

\begin{tabular}{|c|c|c|c|c|c|c|c|c|c|}
\hline Study & Weaning age, $d$ & Initial BW, kg & Growth promoters ${ }^{\mathrm{a}}$ & $\begin{array}{l}\text { Duration of } \\
\text { feeding, } d \text { of age }\end{array}$ & Dietary CP, \% & Lactose level, \% & $\begin{array}{l}\text { ADFI, } \\
\text { g/d }\end{array}$ & $\begin{array}{l}\text { ADG, } \\
g / d\end{array}$ & G:F \\
\hline $\begin{array}{l}\text { Nessmith } \\
\text { et al. [43] }\end{array}$ & 19 & 5.3 & $+(\mathrm{AGP})$ & $19-26$ & - & $0,20,40$ & $\begin{array}{l}196 \\
215 \\
220\end{array}$ & $\begin{array}{l}234 \\
252 \\
246\end{array}$ & $\begin{array}{l}1.19, \\
1.18, \\
1.15\end{array}$ \\
\hline $\begin{array}{l}\text { Nessmith } \\
\text { et al. [43] }\end{array}$ & 19 & 5.3 & $+(\mathrm{AGP})$ & $26-33$ & - & $0,20,40$ & $\begin{array}{l}310 \\
301 \\
300\end{array}$ & $\begin{array}{l}328 \\
322 \\
322\end{array}$ & $\begin{array}{l}1.06, \\
1.08, \\
1.08\end{array}$ \\
\hline $\begin{array}{l}\text { Nessmith } \\
\text { et al. [60] }\end{array}$ & 10 & 3.7 & $+(\mathrm{AGP})$ & $10-15$ & - & $0,20,40$ & $\begin{array}{l}87 \\
104 \\
107\end{array}$ & $\begin{array}{l}109 \\
119 \\
125\end{array}$ & $\begin{array}{l}1.21, \\
1.16, \\
1.15\end{array}$ \\
\hline $\begin{array}{l}\text { Nessmith } \\
\text { et al. [60] }\end{array}$ & 10 & 3.7 & $+(\mathrm{AGP})$ & $15-20$ & - & $0,20,40$ & $\begin{array}{l}152 \\
180 \\
180\end{array}$ & $\begin{array}{l}156 \\
181 \\
184\end{array}$ & $\begin{array}{l}1.02, \\
1.00, \\
1.03\end{array}$ \\
\hline $\begin{array}{l}\text { Tran et al. } \\
{[54]}\end{array}$ & 20 & 6.07 & $+(\mathrm{AGP})$ & $20-27$ & $22 \%$ & 0,20 & $\begin{array}{l}113 \\
117\end{array}$ & 50,71 & $\begin{array}{l}0.44, \\
0.61\end{array}$ \\
\hline $\begin{array}{l}\text { Tran et al. } \\
{[54]}\end{array}$ & 20 & 6.07 & $+(\mathrm{AGP})$ & $27-34$ & $22 \%$ & 0,20 & $\begin{array}{l}340 \\
390\end{array}$ & $\begin{array}{l}286 \\
310\end{array}$ & $\begin{array}{l}0.84, \\
0.82\end{array}$ \\
\hline $\begin{array}{l}\text { Tran et al. } \\
{[54]}\end{array}$ & 20 & 6.07 & $+(\mathrm{AGP})$ & $34-48$ & $22 \%$ & 0,15 & $\begin{array}{l}765 \\
828\end{array}$ & $\begin{array}{l}545 \\
567\end{array}$ & $\begin{array}{l}0.71, \\
0.68\end{array}$ \\
\hline $\begin{array}{l}\text { Tran et al. } \\
\text { [54] }\end{array}$ & 20 & 6.07 & $+(\mathrm{AGP})$ & $48-55$ & $22 \%$ & 0,5 & $\begin{array}{l}1044 \\
1072\end{array}$ & $\begin{array}{l}717 \\
719\end{array}$ & $\begin{array}{l}0.69, \\
0.67\end{array}$ \\
\hline $\begin{array}{l}\text { O' } \\
\text { Doherty } \\
\text { et al. [47] }\end{array}$ & 24 & 7.6 & - & $24-31$ & $20 \%$ & 15,25 & $\begin{array}{l}242 \\
239\end{array}$ & $\begin{array}{l}100 \\
146\end{array}$ & $\begin{array}{l}0.41 \\
0.59\end{array}$ \\
\hline $\begin{array}{l}\text { O' } \\
\text { Doherty } \\
\text { et al. [47] }\end{array}$ & 24 & 7.6 & - & $31-38$ & $20 \%$ & 15,25 & $\begin{array}{l}415 \\
450\end{array}$ & $\begin{array}{l}302, \\
325\end{array}$ & $\begin{array}{l}0.75 \\
0.73\end{array}$ \\
\hline $\begin{array}{l}\text { O' } \\
\text { Doherty } \\
\text { et al. [47] }\end{array}$ & 24 & 7.6 & - & $38-49$ & $20 \%$ & 15,25 & $\begin{array}{l}682 \\
683\end{array}$ & $\begin{array}{l}438 \\
388\end{array}$ & $\begin{array}{l}063 \\
0.57\end{array}$ \\
\hline $\begin{array}{l}\text { O' } \\
\text { Doherty } \\
\text { et al. [46] }\end{array}$ & 22,28 & $7,7.7$ & $+(\mathrm{AGP})$ & $25-32$ & $22 \%$ & $0,17.5,35$ & $\begin{array}{l}209 \\
223 \\
224\end{array}$ & $\begin{array}{l}72, \\
122, \\
163\end{array}$ & $\begin{array}{l}0.34, \\
0.55, \\
073\end{array}$ \\
\hline $\begin{array}{l}\text { O' } \\
\text { Doherty } \\
\text { et al. [46] }\end{array}$ & 22,28 & $7,7.7$ & $+(\mathrm{AGP})$ & $32-42$ & $22 \%$ & $0,17.5,35$ & $\begin{array}{l}363 \\
403 \\
404\end{array}$ & $\begin{array}{l}180 \\
281 \\
265\end{array}$ & $\begin{array}{l}0.46, \\
0.66, \\
0.61\end{array}$ \\
\hline $\begin{array}{l}\mathrm{O}^{\prime} \\
\text { Doherty } \\
\text { et al. [46] }\end{array}$ & 22,28 & $7,7.7$ & $+(\mathrm{AGP})$ & $42-50$ & $22 \%$ & $0,17.5,35$ & $\begin{array}{l}558 \\
660 \\
704\end{array}$ & $\begin{array}{l}267 \\
424 \\
478\end{array}$ & $\begin{array}{l}0.47, \\
0.64, \\
0.65\end{array}$ \\
\hline $\begin{array}{l}\text { Kim et al. } \\
{[48]}\end{array}$ & 21 & 6.94 & $+(\mathrm{AGP})$ & $21-28$ & $21 \%$ & 15,25 & $\begin{array}{l}298 \\
335\end{array}$ & $\begin{array}{l}247 \\
289\end{array}$ & $\begin{array}{l}0.83, \\
0.86\end{array}$ \\
\hline $\begin{array}{l}\text { Kim et al. } \\
{[48]}\end{array}$ & 28 & 9.57 & $+(\mathrm{AGP})$ & $28-35$ & $21 \%$ & 15,25 & $\begin{array}{l}542 \\
579\end{array}$ & $\begin{array}{l}423 \\
461\end{array}$ & $\begin{array}{l}0.78, \\
0.79\end{array}$ \\
\hline $\begin{array}{l}\text { Pierce } \\
\text { et al. [49] }\end{array}$ & 24 & 7.1 & - & $24-31$ & $20 \%$ & $6.5,17,27.5$ & $\begin{array}{l}189 \\
192 \\
196\end{array}$ & $\begin{array}{l}73,80 \\
80\end{array}$ & $\begin{array}{l}0.39, \\
0.42, \\
0.41\end{array}$ \\
\hline $\begin{array}{l}\text { Pierce } \\
\text { et al. [49] }\end{array}$ & 24 & 7.1 & - & $31-38$ & $20 \%$ & $6.5,17,27.5$ & $\begin{array}{l}335 \\
329 \\
341\end{array}$ & $\begin{array}{l}175 \\
224 \\
212\end{array}$ & $\begin{array}{l}0.52, \\
0.68, \\
0.63\end{array}$ \\
\hline $\begin{array}{l}\text { Pierce } \\
\text { et al. [49] }\end{array}$ & 24 & 7.1 & - & $38-45$ & $20 \%$ & $6.5,17,27.5$ & $\begin{array}{l}512, \\
511 \\
567\end{array}$ & $\begin{array}{l}292 \\
322 \\
358\end{array}$ & $\begin{array}{l}0.61, \\
0.68, \\
0.66\end{array}$ \\
\hline $\begin{array}{l}\text { Pierce } \\
\text { et al. [49] }\end{array}$ & 24 & 7.1 & - & $45-52$ & $20 \%$ & $6.5,17,27.5$ & $\begin{array}{l}652 \\
677 \\
737\end{array}$ & $\begin{array}{l}399 \\
461 \\
487\end{array}$ & $\begin{array}{l}0.56, \\
0.65, \\
0.63\end{array}$ \\
\hline Pierce & 24 & 6 & - & $24-31$ & $22 \%$ & $17,29.5$ & 254 & 99 & 0.39 \\
\hline
\end{tabular}


Table 1 Summary of studies evaluating the effect of dietary lactose concentration on growth performance of weanling pigs used in the meta-analysis (Continued)

\begin{tabular}{|c|c|c|c|c|c|c|c|c|c|}
\hline Study & Weaning age, $d$ & Initial BW, kg & Growth promoters ${ }^{\mathrm{a}}$ & $\begin{array}{l}\text { Duration of } \\
\text { feeding, } d \text { of age }\end{array}$ & Dietary CP, \% & Lactose level, \% & $\begin{array}{l}\text { ADFI, } \\
\text { g/d }\end{array}$ & $\begin{array}{l}A D G \\
g / d\end{array}$ & G:F \\
\hline et al. [33] & & & & & & & 323 & 179 & 0.56 \\
\hline $\begin{array}{l}\text { Pierce } \\
\text { et al. [33] }\end{array}$ & 24 & 6 & - & $31-38$ & $22 \%$ & $17,29.5$ & $\begin{array}{l}441 \\
507\end{array}$ & $\begin{array}{l}290 \\
331\end{array}$ & $\begin{array}{l}0.66, \\
0.65\end{array}$ \\
\hline $\begin{array}{l}\text { Pierce } \\
\text { et al. [33] }\end{array}$ & 24 & 6 & - & $38-45$ & $22 \%$ & $17,29.5$ & $\begin{array}{l}757 \\
876\end{array}$ & $\begin{array}{l}452 \\
527\end{array}$ & $\begin{array}{l}0.6 \\
0.61\end{array}$ \\
\hline $\begin{array}{l}\text { Mahan } \\
\text { and } \\
\text { Newton } \\
\text { [50] }\end{array}$ & 23 & 6.5 & $+(\mathrm{AGP})$ & $23-37$ & - & $0,22.5,35.0$ & $\begin{array}{l}301 \\
338 \\
312\end{array}$ & $\begin{array}{l}243 \\
278 \\
258\end{array}$ & $\begin{array}{l}0.81, \\
0.82, \\
0.82\end{array}$ \\
\hline $\begin{array}{l}\text { Bertol } \\
\text { et al. [51] }\end{array}$ & 21 & & $+(\mathrm{AGP})$ & $21-35$ & $20 \%$ & $0,7,14,21$ & $\begin{array}{l}240 \\
280 \\
300 \\
334\end{array}$ & $\begin{array}{l}142 \\
186 \\
191 \\
219\end{array}$ & $\begin{array}{l}0.55, \\
0.65, \\
0.63, \\
0.65\end{array}$ \\
\hline $\begin{array}{l}\text { Bertol } \\
\text { et al. [51] }\end{array}$ & 19 & 6.3 & $+(\mathrm{AGP})$ & $19-26$ & - & $\begin{array}{l}10,15,20,25,30, \\
35\end{array}$ & $\begin{array}{l}190 \\
207 \\
202 \\
214 \\
225 \\
194\end{array}$ & $\begin{array}{l}84,99 \\
103 \\
114 \\
130 \\
103\end{array}$ & $\begin{array}{l}0.42, \\
0.48, \\
0.51, \\
0.53, \\
0.58, \\
0.53\end{array}$ \\
\hline $\begin{array}{l}\text { Bertol } \\
\text { et al. [51] }\end{array}$ & 19 & 6.3 & $+(\mathrm{AGP})$ & $26-33$ & - & $\begin{array}{l}10,15,20,25,30, \\
35\end{array}$ & $\begin{array}{l}401 \\
412 \\
423 \\
428 \\
421 \\
409\end{array}$ & $\begin{array}{l}346 \\
349 \\
370 \\
360 \\
359 \\
330\end{array}$ & $\begin{array}{l}0.86, \\
0.85 \\
0.88, \\
0.84 \\
0.86 \\
0.83\end{array}$ \\
\hline $\begin{array}{l}\text { Bertol } \\
\text { et al. [51] }\end{array}$ & 19 & 6.3 & $+(\mathrm{AGP})$ & $26-33$ & - & $\begin{array}{l}7,12,17,22,27, \\
32\end{array}$ & $\begin{array}{l}421 \\
397 \\
426 \\
448 \\
427 \\
449\end{array}$ & $\begin{array}{l}278 \\
285 \\
315 \\
335 \\
317 \\
331\end{array}$ & $\begin{array}{l}0.66, \\
0.72, \\
0.74, \\
0.75, \\
0.74, \\
0.74\end{array}$ \\
\hline $\begin{array}{l}\text { Bertol } \\
\text { et al. [51] }\end{array}$ & 19 & 6.3 & $+(\mathrm{AGP})$ & $33-40$ & - & $\begin{array}{l}7,12,17,22,27, \\
32\end{array}$ & $\begin{array}{l}700 \\
690 \\
736 \\
718 \\
705 \\
718\end{array}$ & $\begin{array}{l}507 \\
509 \\
525 \\
514 \\
529 \\
540\end{array}$ & $\begin{array}{l}0.72, \\
0.74, \\
0.71 \\
0.70 \\
0.75 \\
0.75\end{array}$ \\
\hline $\begin{array}{l}\text { Bertol } \\
\text { et al. [51] }\end{array}$ & 19 & 6.3 & $+(\mathrm{AGP})$ & $40-54$ & - & $0,5,10,15,20$ & $\begin{array}{l}1095 \\
1104 \\
1106 \\
1124 \\
1135\end{array}$ & $\begin{array}{l}657 \\
685 \\
678 \\
695 \\
686\end{array}$ & $\begin{array}{l}0.6, \\
0.62, \\
0.61 \\
0.62 \\
0.60\end{array}$ \\
\hline $\begin{array}{l}\text { Molino } \\
\text { et al. [58] }\end{array}$ & 21 & 6.1 & $+(\mathrm{AGP})$ & $21-35$ & $21 \%$ & $0,4,8,12$ & $\begin{array}{l}250 \\
308 \\
280 \\
306\end{array}$ & $\begin{array}{l}195 \\
244 \\
220 \\
248\end{array}$ & $\begin{array}{l}0.78, \\
0.78, \\
0.78, \\
0.8\end{array}$ \\
\hline $\begin{array}{l}\text { Cromwell } \\
\text { et al. [59] }\end{array}$ & 20 & 6.2 & $+(\mathrm{AGP})$ & $30-37$ & $22 \%$ & $0,2.5,5,7.5,10$ & $\begin{array}{l}613 \\
618 \\
643 \\
646 \\
648\end{array}$ & $\begin{array}{l}467 \\
467 \\
503 \\
505 \\
506\end{array}$ & $\begin{array}{l}0.76, \\
0.76, \\
0.78, \\
0.78 \\
0.78\end{array}$ \\
\hline $\begin{array}{l}\text { Cromwell } \\
\text { et al. [59] }\end{array}$ & 20 & 6.2 & $+(\mathrm{AGP})$ & $37-44$ & $22 \%$ & $0,2.5,5,7.5,10$ & $\begin{array}{l}833 \\
830 \\
826 \\
860 \\
856\end{array}$ & $\begin{array}{l}597 \\
600 \\
592 \\
610 \\
610\end{array}$ & $\begin{array}{l}0.72, \\
0.73, \\
0.73, \\
0.72, \\
0.72\end{array}$ \\
\hline $\begin{array}{l}\text { Pierce } \\
\text { et al. [61] }\end{array}$ & 21 & 7.6 & - & $33-45$ & $16 \%$ & $12.5,21.5$ & $\begin{array}{l}600 \\
600\end{array}$ & $\begin{array}{l}270 \\
300\end{array}$ & $\begin{array}{l}0.45 \\
0.5\end{array}$ \\
\hline
\end{tabular}


Table 1 Summary of studies evaluating the effect of dietary lactose concentration on growth performance of weanling pigs used in the meta-analysis (Continued)

\begin{tabular}{|c|c|c|c|c|c|c|c|c|c|}
\hline Study & Weaning age, $d$ & Initial BW, kg & Growth promoters $^{\mathrm{a}}$ & $\begin{array}{l}\text { Duration of } \\
\text { feeding, } d \text { of age }\end{array}$ & Dietary CP, \% & Lactose level, \% & $\begin{array}{l}\text { ADFI, } \\
\text { g/d }\end{array}$ & $\begin{array}{l}\text { ADG, } \\
\text { g/d }\end{array}$ & G:F \\
\hline $\begin{array}{l}\text { Pierce } \\
\text { et al. [61] }\end{array}$ & 21 & 7.6 & - & $45-54$ & $16 \%$ & $12.5,21.5$ & $\begin{array}{l}920 \\
870\end{array}$ & $\begin{array}{l}520 \\
490\end{array}$ & $\begin{array}{l}0.55, \\
0.51\end{array}$ \\
\hline $\begin{array}{l}\text { Pierce } \\
\text { et al. [61] }\end{array}$ & 21 & 7.6 & - & $33-45$ & $18.5 \%$ & $12.5,21.5$ & $\begin{array}{l}650 \\
640\end{array}$ & $\begin{array}{l}400 \\
380\end{array}$ & $\begin{array}{l}0.61, \\
0.59\end{array}$ \\
\hline $\begin{array}{l}\text { Pierce } \\
\text { et al. [61] }\end{array}$ & 21 & 7.6 & - & $45-54$ & $18.5 \%$ & $12.5,21.5$ & $\begin{array}{l}1070 \\
890\end{array}$ & $\begin{array}{l}620 \\
590\end{array}$ & $\begin{array}{l}0.56, \\
0.66\end{array}$ \\
\hline $\begin{array}{l}\text { Pierce } \\
\text { et al. [61] }\end{array}$ & 21 & 7.6 & - & $33-45$ & $21 \%$ & $12.5,21.5$ & $\begin{array}{l}600 \\
700\end{array}$ & $\begin{array}{l}400 \\
400\end{array}$ & $\begin{array}{l}0.67, \\
0.59\end{array}$ \\
\hline $\begin{array}{l}\text { Pierce } \\
\text { et al. [61] }\end{array}$ & 21 & 7.6 & - & $45-54$ & $21 \%$ & $12.5,21.5$ & $\begin{array}{l}970 \\
1100\end{array}$ & $\begin{array}{l}570 \\
700\end{array}$ & $\begin{array}{l}0.6 \\
0.63\end{array}$ \\
\hline $\begin{array}{l}\mathrm{O}^{\prime} \\
\text { Connell } \\
\text { et al. [62] }\end{array}$ & 24 & 6 & - & $24-31$ & $20 \%$ & $17,27.5$ & $\begin{array}{l}190 \\
212\end{array}$ & $\begin{array}{l}104 \\
121\end{array}$ & $\begin{array}{l}0.55, \\
0.57\end{array}$ \\
\hline $\begin{array}{l}\text { O' } \\
\text { Connell } \\
\text { et al. [62] }\end{array}$ & 24 & 6 & - & $31-38$ & $20 \%$ & $17,27.5$ & $\begin{array}{l}434, \\
462\end{array}$ & $\begin{array}{l}364 \\
394\end{array}$ & $\begin{array}{l}0.84, \\
0.85\end{array}$ \\
\hline $\begin{array}{l}\text { O' } \\
\text { Connell } \\
\text { et al. [62] }\end{array}$ & 24 & 6 & - & $38-49$ & $20 \%$ & $17,27.5$ & $\begin{array}{l}615 \\
691\end{array}$ & $\begin{array}{l}422, \\
475\end{array}$ & $\begin{array}{l}0.69, \\
0.69\end{array}$ \\
\hline $\begin{array}{l}\text { O' } \\
\text { Connell } \\
\text { et al. [62] }\end{array}$ & 24 & 6 & - & $49-57$ & $20 \%$ & $17,27.5$ & $\begin{array}{l}870 \\
883\end{array}$ & $\begin{array}{l}561 \\
538\end{array}$ & $\begin{array}{l}0.65, \\
0.61\end{array}$ \\
\hline
\end{tabular}

${ }^{a}+$ indicates the use of in feed antibiotic growth promotors (AGP) and/or pharmacological dose of $\mathrm{ZnO}$ (>1500 ppm) and/or pharmacological dose of CuSO ${ }_{4}(>$ $150 \mathrm{ppm}$ ). ADFI Average daily feed intake, $A D G$ Average daily gain, BW Body weight, CP Crude protein, G:F The ratio of weight gain to feed intake

Models were created for 3 growth phases: $d 0-7$, d 7-14, and d 14-35 post-wean to reflect the physiological lactase activity of piglets post-wean as well as commercial phase feeding practices [30]. Dietary lactose concentration was used as the sole predictor variable in the models. A total of 12 studies containing 38 experimental diets varying in dietary lactose levels, an average weaning age of $22 \mathrm{~d}$ and initial BW of $6.56 \mathrm{~kg}$ were used in model development for $\mathrm{d} 0-7$ post-wean. Ten studies with a total of 40 experimental diets, an average start age of $26 \mathrm{~d}$ and initial BW of $7.06 \mathrm{~kg}$ were used in model development for $\mathrm{d} 7-$ 14 post-wean. Finally, for d 14-35 post-wean, 9 studies with a total of 54 experimental diets, an average start age of $38 \mathrm{~d}$ and initial BW of $10.96 \mathrm{~kg}$ were used in model development. In the models, different weaning ages were not included as a covariate because there was not large variation in weaning age amongst studies. There were 2 data points with weaning age of 10 and 4 data points with weaning age of 28 , while the rest of the data points (40) had weaning ages from 19 to $24 \mathrm{~d}$. Therefore, more data is needed with an early weaning age and much later weaning ages to use weaning age in the model. The selected data were then subjected to a statistical metaregression based on a mixed model methodology [63]. Accordingly, different studies were treated as random effects, whereas dietary lactose level was considered a fixed effect. For the predictor variable of dietary lactose levels, the following model was used:

$$
\mathrm{Y}_{\mathrm{ij}}=\mathrm{B}_{0}+\mathrm{B}_{1} \mathrm{X}_{\mathrm{ij}}+\mathrm{B}_{2} \mathrm{X}_{\mathrm{ij}}^{2}+\mathrm{s}_{\mathrm{i}}+\mathrm{b}_{\mathrm{i}} \mathrm{X}_{\mathrm{ij}}+\mathrm{e}_{\mathrm{ij}}
$$

where $Y_{\mathrm{ij}}=$ dependent variable of ADFI, ADG or G:F, $B_{0}=$ overall intercept across all studies, $B_{1}=$ linear regression coefficient of $Y$ on $X$ (fixed effect), $B_{2}=$ quadratic regression coefficient of $Y$ on $X$ (fixed effect), $X_{i j}=$ value of the predictor variable (dietary lactose level), $s_{i}=$ random effect of study $i, b_{i}=$ random effect of study $i$ on the regression coefficient of $Y$ on $X$ in study $i$, and $e_{i j}=$ the unexplained residual error. The linear or quadratic model that resulted in a slope different $(P<0.10)$ from 0 was used. The model that had the greater $\mathrm{R}^{2}$ and lower RMSE and AIC was used when both linear and quadratic models were different from 0 .

Model predicted pig ADFI, ADG and G:F responses to increased dietary lactose levels are presented in Table 2. Pig ADG $(P<0.10)$ and G:F $(P<0.01)$ linearly increased as lactose concentration increased in diets fed to weanling pigs from $\mathrm{d}$ 0-7 post-wean. The slope of the models to predict ADFI was not different from 0 and this was most probably due to the large variation in ADFI across studies from d 0-7 post-wean. This may be a result of 
Table 2 Meta-regression of growth performance in weanling pigs to changes in different concentrations of dietary lactose

\begin{tabular}{|c|c|c|c|c|c|c|c|c|c|}
\hline Items & $\begin{array}{l}\text { Response } \\
\text { variable }\end{array}$ & $\begin{array}{l}\text { Linear } \\
\mathrm{R}^{2}\end{array}$ & $\begin{array}{l}\text { Linear } \\
\text { RMSE }\end{array}$ & $\begin{array}{l}\text { Linear } \\
\text { AIC }\end{array}$ & $\begin{array}{l}\text { Linear } P \text { - } \\
\text { value }\end{array}$ & $\begin{array}{l}\text { Quadratic } \\
\mathrm{R}^{2}\end{array}$ & $\begin{array}{l}\text { Quadratic } \\
\text { RMSE }\end{array}$ & $\begin{array}{l}\text { Quadratic } \\
\text { AIC }\end{array}$ & $\begin{array}{l}\text { Quadratic } P \text { - } \\
\text { value }\end{array}$ \\
\hline \multicolumn{10}{|c|}{ d 0-7 post-wean } \\
\hline $\begin{array}{l}\text { ADFI, g/ } \\
\text { d }\end{array}$ & Lactose level, \% & 0.800 & 66.66 & 235.5 & 0.313 & 0.802 & 68.85 & 237.6 & 0.504 \\
\hline $\begin{array}{l}\text { ADG, g/ } \\
d\end{array}$ & Lactose level, \% & 0.901 & 48.35 & 226.5 & 0.069 & 0.903 & 49.63 & 229.1 & 0.259 \\
\hline $\mathrm{G}: \mathrm{F}$ & Lactose level, \% & 0.995 & 0.025 & 15.01 & 0.001 & 0.996 & 0.135 & 30.8 & 0.141 \\
\hline \multicolumn{10}{|c|}{ d 7-14 post-wean } \\
\hline $\begin{array}{l}\text { ADFI, g/ } \\
\text { d }\end{array}$ & Lactose level, \% & 0.992 & 13.38 & 580.0 & 0.001 & 0.993 & 12.69 & 593.2 & 0.094 \\
\hline $\begin{array}{l}\text { ADG, g/ } \\
d\end{array}$ & Lactose level, \% & 0.963 & 24.73 & 603.3 & 0.004 & 0.973 & 21.86 & 158.0 & 0.031 \\
\hline $\mathrm{G}: \mathrm{F}$ & Lactose level, \% & 0.887 & 0.062 & 37.6 & 0.435 & 0.904 & 0.093 & 0.058 & 0.093 \\
\hline \multicolumn{10}{|c|}{ d 14-35 post-wean } \\
\hline $\begin{array}{l}\text { ADFI, g/ } \\
d\end{array}$ & Lactose level, \% & 0.388 & 167.2 & 534.6 & 0.830 & 0.388 & 169.4 & 534.5 & 0.844 \\
\hline $\begin{array}{l}\text { ADG, g/ } \\
d\end{array}$ & Lactose level, \% & 0.520 & 98.15 & 496.3 & 0.726 & 0.522 & 99.32 & 497.1 & 0.696 \\
\hline $\mathrm{G}: \mathrm{F}$ & Lactose level, \% & 0.705 & 0.05 & -49.2 & 0.723 & 0.708 & 0.051 & -33.5 & 0.514 \\
\hline
\end{tabular}

an immature digestive tract and lack of appetite for solid feed due to large adaptations and high levels of stress in pigs in the first week after weaning. This can potentially be ameliorated by later weaning (e.g. $35 \mathrm{~d}$ ) and/or the application of a pre-starter diet.

Pigs begin to increase feed intake 1 week after weaning because the gastrointestinal tract begins to adapt to the solid diets. Accordingly, linear and quadratic models to predict ADFI, ADG and G:F of weanling pigs from d 714 post-wean were significantly different from 0 . Modeled results indicated that ADG was greatest with an inclusion of $15 \%$ lactose, but $30 \%$ lactose optimized ADFI. The model also indicated ADG of pigs was reduced when pigs were fed levels of dietary lactose greater than $15 \%$ during $\mathrm{d} 7-14$ post-wean. It may be inferred that excessive fermentation of lactose may occur over 15\% dietary lactose fed to pigs in d 7-14 post-wean because $30 \%$ lactose optimized ADFI, but 15\% lactose optimized ADG. Excessive fermentation of lactose may result in osmotic imbalance in the intestinal tract and may lead to diarrhea and reduced ADG of pigs.

There was no linear $(P>0.05)$ or quadratic $(P>$ $0.05)$ response of dietary lactose levels on growth performance of pigs from d 14-35 post-wean. Lactose most probably had a limited effect on performance in this phase due to low lactase activity. The negative ADFI, ADG, and G:F responses to increased levels of dietary lactose, although not significant, indicated that lactose may even be detrimental to growth performance of pigs in d 14-35 post-wean. We hypothesize that this could be due to reduced lactase activity, increased feed intake, and excessive fermentation of lactose as pigs get older.

In summary, dietary lactose levels produced different responses on ADFI, ADG and G:F of pigs during different feeding phases, which appear to be associated with the reported lactase activity as pigs get older. In the first week post-wean, there was no limitation of lactose based on responses of ADG and G:F in weanling pigs. This indicates that lactase activity is sufficient to digest high quantities of lactose from d 0-7 post-wean. However, pig ADG decreased when pigs were fed levels of dietary lactose greater than $15 \%$ from $d$ 7-14 post-wean. This may be caused by excessive intake and fermentation of lactose due to reduced lactase activity. Therefore, results of the meta-analysis indicate that high lactose levels can be fed to pigs in the early period after weaning, but a decreased inclusion level of lactose should be considered to avoid negative responses of pig ADFI and ADG in later periods. A quadratic regression model was applied (Fig. 3) to determine a potential dietary lactose requirement for weanling pigs based on the literature. The model indicated that the level of dietary lactose to optimize ADG of weanling pigs from d $0-7,7-14$, and $14-35$ post-wean was $20 \%, 15 \%$, and 0 , respectively, for piglets with an average initial BW of $6.56 \mathrm{~kg}$ and weaned at $22 \mathrm{~d}$ of age. The recommendations for pigs from $\mathrm{d} 0$ 7 and $d$ 7-14 post-wean from the present meta-analysis are similar to the dietary lactose recommendations provided by Mahan et al. [52] and Cromwell et al. [59]. However, both Mahan et al. [52] and Cromwell et al. [59] have much higher lactose recommendations for pigs 


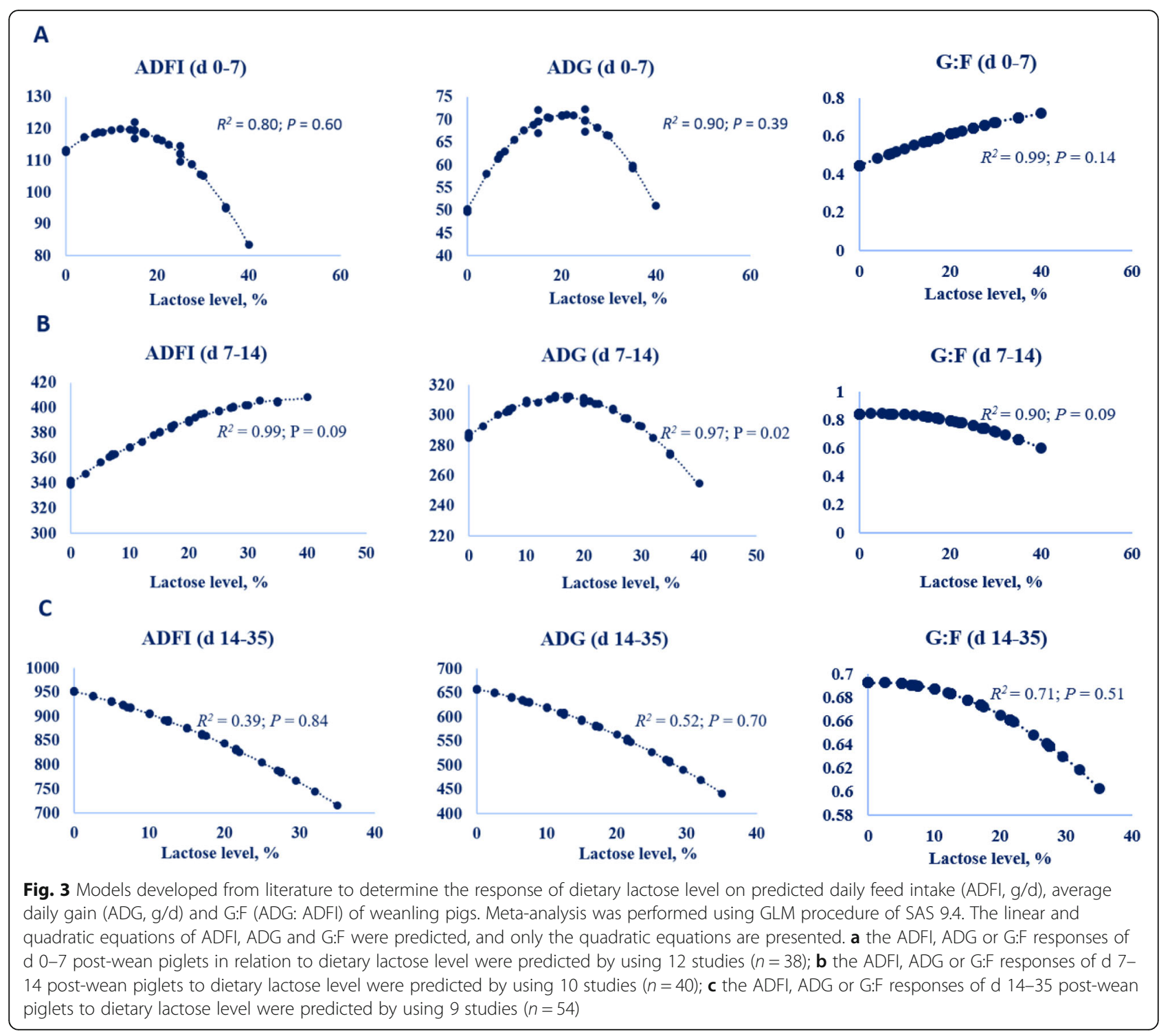

from d 14-35 post-wean, whereas our results recommend the addition of no lactose in this later phase of the nursery. Some previous studies reported that the positive effects of dietary lactose supplementation on growth performance of weanling pigs was dependent on the cleanliness of the environment $[1,59]$ and this could be a reason for the discrepancy in recommendations amongst many other factors.

Therefore, it was hypothesized that pigs fed diets containing antibiotic growth promotors (AGP; antibiotics, pharmacological levels of $\mathrm{ZnO}$, and/or supra-nutritional levels of $\mathrm{Cu}$ ) would have a cleaner environment and, therefore, a different response to dietary lactose levels. This hypothesis was tested using the same meta-analysis technique in our review. A total of 6 studies containing 54 experimental diets with AGP addition and 4 studies containing 18 experimental diets without AGP were each separately used to develop models to determine the quadratic effect of lactose level on growth performance of pigs during d 0-14 post-wean. Further, for d 14-35 post-wean, 4 studies containing 28 experimental diets with AGP addition and 5 studies containing 26 experimental diets without AGP were each separately used to develop models to determine the quadratic effect of lactose level on growth performance of pigs (Table 1). The model determined that using diets which contained AGP numerically decreased the positive ADFI, ADG and G:F responses of pigs fed greater levels of lactose in diets fed to pigs from d $0-14$ and d 14-35 post-wean (Supplementary Figure 1). This may indicate that the prebiotic effect of lactose in weanling pigs may be minimized by the use of AGP. These results are based on the numerical comparison of slopes of the 2 quadratic models (+AGP vs. -AGP) and further interpretation and use of 
the results are cautioned due to the fact that the slopes of all models were not different $(P>0.10)$ from 0 . The observations for no significant responses of dietary lactose levels on pig ADFI and ADG is most probably due to an insufficient number of data points (i. e. references containing AGP versus references without AGP). Therefore, a larger dataset is warranted to further investigate the potential interaction between AGP and the prebiotic role lactose may play in weanling pig nutrition, especially in farms that have poor pig hygiene and health.

\section{Interactions between lactose and other nutrients or feed additives on growth performance}

Interactions have been reported on performance of weanling pigs between lactose level and dietary nutrients or feed additives, such as $\beta$-glucanase, organic acids, antibiotics, protein and fermentable dietary fiber. For example, lactose levels above $7.5 \%$ in diets fed from $\mathrm{d} 0-7$ post-wean and above $6.3 \%$ lactose in diets fed from $\mathrm{d} 7-$ 14 post-wean resulted in improved ADG, but the combination of an acidifier and high lactose did not further alter growth performance [64]. Lynch et al. [65] found an interaction between crude protein (CP) and lactose concentration that resulted in a positive effect of $23 \%$ lactose on performance of piglets (BW of $7.4 \mathrm{~kg}$ and weaned at 24 days old) fed diets containing $20 \% \mathrm{CP}$ compared with pigs fed diets containing 5\% lactose and $20 \% \mathrm{CP}$, but no positive effects of lactose concentration on growth performance of weanling pigs when fed a $16 \%$ $\mathrm{CP}$ diet. Furthermore, Pierce et al. [61] indicated growth performance of piglets with an initial age of $35 \mathrm{~d}$ and fed a high lactose diet $(21.5 \%$ vs. $12.5 \%)$ was positively related to protein level of diets (16\%, $18.5 \%$ and $21 \%)$. This may be partially explained by the relative effects of high lactose on improved diet palatability and thereby increased ADFI, which resulted in a larger quantity of protein consumed.

The inclusion of $60 \mathrm{mg} / \mathrm{kg}$ avilamycin and inulin in a diet with $17.5 \%$ lactose fed for 2 weeks post-wean resulted in a proportional improvement in ADG of piglets (initial BW of $6 \mathrm{~kg}$ ). No positive effects were observed, however, when pigs were fed a diet containing $29.5 \%$ lactose, $60 \mathrm{mg} / \mathrm{kg}$ avilamycin and inulin [49]. The lack of pig growth performance response to high dietary lactose in these studies may indicate that the addition of antibiotics or dietary fiber may improve intestinal health similar to a high concentration of lactose, resulting in a decreased recommended level of dietary lactose. This result is in support of our interpretation of a limited model created to compare the use of lactose in diets with and without AGP (Supplementary Figure 1).

Weanling pigs (initial BW of $6 \mathrm{~kg}$ and $24 \mathrm{~d}$ of age) fed a barley-based diet containing $27.5 \%$ dietary lactose had decreased ADG compared with piglets fed the diet with
$17 \%$ lactose in the first week after weaning, but the result of the effect of dietary lactose level on growth performance was reversed when piglets were fed a wheatbased diet [62]. This interaction on performance of pigs between lactose supplementation and cereal type may be explained because the $\beta$-glucan in barley may potentially replace a part of the lactose as a prebiotic substrate for fermentation by gastrointestinal microbiota. In a previous study, the inclusion of $\beta$-glucanase to a diet containing $17 \%$ lactose improved ADG, G:F and nutrient digestibility in piglets with an initial BW of $6.5 \mathrm{~kg}$ and weaned at 24 days old, but no positive effects on ADG and G:F were observed when $\beta$-glucanase was added to the diet with $27.5 \%$ lactose [66]. This observation was most probably caused by the interaction between dietary lactose and $\beta$-glucanase, which was associated with a greater digestibility of dietary fiber and production of VFA induced by $\beta$-glucanase supplementation, thus minimizing the prebiotic effects of lactose. These results support the use of lactose as a prebiotic for weanling pigs. However, to achieve a consistent and beneficial response, the role of dietary fiber, AGP, and $\beta$-glucanase, amongst other factors, and their interactions must be clearly understood and is subject for further investigation.

\section{Effect of lactose on nutrient digestibility}

Increasing dietary lactose can improve nutrient digestibility because it is an easily digestible and/or fermentable component in the intestine of weanling pigs and this results in a greater apparent digestibility of nutrients $[47,67]$. Another explanation for the positive effect of lactose on nutrient digestibility could be due to lactic acid and VFA produced by lactose fermentation in the stomach which decreases stomach $\mathrm{pH}$, and thereby improves pepsin activity [45]. A combination of $15 \%$ pure lactose and crystalline amino acids added to a weanling pig (initial BW of $6.8 \mathrm{~kg}$ and a weaning age of $23 \mathrm{~d}$ ) diet improved the apparent total tract digestibility (ATTD) of dry matter (DM), gross energy (GE), and nitrogen retention compared with pigs fed a diet containing lactalbumin. Lactalbum was extracted from sow milk and is a primary protein component of whey powder [67]. Furthermore, inclusion of $25 \%$ lactose in the diet increased the ATTD of nutrients and energy by weanling pigs (average initial BW of $7.6 \mathrm{~kg}$ ) compared with pigs fed a diet containing $15 \%$ lactose [47]. The inclusion of $1.5 \%$ inulin improved energy digestibility by pigs (average initial BW of $6.0 \mathrm{~kg}$ ) fed a diet with $15 \%$ lactose, but did not affect the digestibility by pigs fed the high (29.5\%) lactose diet [49]. The negative response of high lactose level with inulin supplementation may be caused by excessive fermentation of lactose and inulin by gut microbiota, resulting in abnormal intestinal osmotic balance. 
Including $27.5 \%$ dietary lactose decreased the nutrient digestibility by weanling pigs fed a barley-based diet compared with a barley-based diet supplemented with only $17 \%$ lactose. In contrast, pigs fed a wheat-based diet saw in no difference in nutrient digestibility whether or not the diets contained $27.5 \%$ or $17 \%$ lactose [62], which may also be caused by more fiber fermentation provided by barley than the wheat-based diet. These results indicate that the effect of dietary lactose on nutrient digestibility may be associated with dietary composition and most probably related to dietary fiber and microbial fermentation potential. Bach Knudsen [68] compared the ileal digestibility of $\beta$-glucan by $40-50 \mathrm{~kg}$ growing pigs fed diets based on rolled oats, oat groats, oat flour, or oat bran and contained either a high lactose (12 to $38 \mathrm{~g}$ lactose $/ \mathrm{kg} \mathrm{DM}$ ) or low lactose content (0 to $1 \mathrm{~g}$ lactose/ $\mathrm{kg} \mathrm{DM})$. Results indicated that the digestibility of $\beta$ glucan in the ileum was $64 \%$ in diets with low lactose and $27 \%$ in diets containing high lactose, which suggested that lactose was preferentially fermented by gastrointestinal microbiota compared to $\beta$-glucan when there was most likely very little intestinal lactase activity in pigs of $40-50 \mathrm{~kg}$. This study provides further evidence that lactose is preferentially fermented by gastrointestinal microbiota compared with other dietary fibers and that this interaction must be accounted for in order to achieve a prebiotic effect with dietary lactose. A prebiotic effect of lactose is when dietary lactose is not digested, rather it is used as a substrate for fermentation by microbes and this elicits a change in microbial populations and metabolites that improve the health of the pig.

\section{Effect of lactose on intestinal morphology}

Pierce et al. [69] reported no difference in villus height and crypt depth of intestinal segments in weanling piglets (mean initial BW of 7.8 and average $21 \mathrm{~d}$ of age) fed diets with $15 \%$ or $33 \%$ lactose, although piglets fed the high lactose diet did have a reduced cecum and colon $\mathrm{pH}$. Furthermore, in the same study, the inclusion of $1.5 \%$ inulin into the $15 \%$ lactose diet resulted in increased villus height, but inulin added to the diet with $33 \%$ lactose had no effect on pig gut morphology. This indicates that an interaction between dietary lactose level and inulin (i.e., fermentable substrates) on intestinal morphology of weanling piglets exists. There were also no differences in intestinal morphology on $\mathrm{d} 0,3$, and 10 post-wean of piglets fed diets containing either $20 \%$ glucose, lactose or starch [1]. This may partly be due to the fact that these pigs were weaned later ( $28 \mathrm{~d}$ of age) and with a heavier BW $(8 \mathrm{~kg})$ than those in most other lactose studies. The addition of 0 or $12 \%$ lactose in the diet did not affect villous height, crypt depth or villous: crypt ratio of weanling pigs in the first 2 weeks post-wean
[58]. This report is not in agreement with the previous study that reported a positive effect of lactose supplementation on intestinal villus height and capacity for nutrient absorption in weanling pigs [51]. Acosta et al. [70] also reported an increased villous height when weanling piglets (average initial BW of $5.2 \mathrm{~kg}$ and $21 \mathrm{~d}$ of age) were fed a diet containing 15\% lactose compared with a diet containing $3 \%$ lactose. Also, a diet with a high lactose to protein ratio improved the villous height of the proximal small intestine in weanling piglets ( $26 \mathrm{~d}$ of age) compared with a low lactose to protein ratio diet [71]. These results indicate that the digestibility of dietary lactose, rather than protein, could provide more digestible energy or a suitable micro-environment for the development of intestinal epithelial cells. Overall, there have been inconsistent effects of dietary lactose on intestinal morphology in weanling pigs. The inconsistency most likely is associated with variations in dietary lactose level, lactase activity of weanling pigs, and dietary composition. Further studies should be conducted to explore the impact of energy supplied versus the prebiotic response derived from lactose digestion by lactase versus fermentation by gastrointestinal microbiota on intestinal morphology and the health of weanling pigs.

\section{Effect of lactose on gastrointestinal microbiota}

Dietary lactose has been shown to have prebiotic effects on microbiota in the gastrointestinal tract of weanling piglets [72]. This is because it may be partially fermented by microbiota to lactic acid and VFA to provide gastric and colonic acidity [68]. Lactic acid and VFA can reduce stomach $\mathrm{pH}$ and create a less favorable environment for pathogens and suppress the growth of $E$. coli, thereby improving the gastrointestinal health of the host [73]. Furthermore, a high concentration of dietary lactose (20\%) increased the relative abundance of lactobacilli and reduced $E$. coli in the large intestine of piglets in the first week post-wean [54]. The inclusion of lactose in diets for weanling pigs increased the population of total bacteria in the colon and Lactobacillus abundance in the ileum and colon per gram of digesta content [74]. The inclusion of either $25 \%$ or $15 \%$ lactose decreased the counts of $E$. coli in the feces of piglets after weaning [47]. The inclusion of lactose in the diet resulted in a greater diversity and abundance of the beneficial bacteria in the intestine of weanling pigs in the first 2 weeks post-wean, and the Lactobacillus richness increased with the addition of $8 \%$ lactose, although the population of total bacteria decreased when piglets were fed $12 \%$ lactose [64]. In addition, the supplementation of Bifidobacterium increased lactose fermentation and lactic acid production, but not acetate, helping to regulate the microbial composition in the gastrointestinal tract of piglets [75]. Overall, our findings in this review indicate 
that dietary lactose has a prebiotic effect on the gastrointestinal microbiota. This mode of action of lactose must be further explored to enhance weanling pig gastrointestinal health in light of new legislation and consumer demands limiting the use of AGP, pharmacological levels of $\mathrm{ZnO}$, and supra-nutritional levels of $\mathrm{Cu}$. However, a high lactose supplementation and/or intake may result in excessive fermentation of lactose and this could be detrimental to growth performance as hypothesized from our meta-analysis as well as increase risk of post-weaning diarrhea when weaning stress and herd hygiene and health are not good. Thus, the prebiotic action of lactose fed to weanling pigs needs to be further explored.

\section{Lactose in growing-finishing pig diets}

Few studies have reported effects of dietary lactose fed to growing-finishing pigs from 25 to $130 \mathrm{~kg} \mathrm{BW}$. This is mostly because adult pigs have a high capacity for digesting other carbohydrates which are typically less costly than lactose [76]. A previous study reported a reduced ATTD of nutrients in adult pigs offered a diet containing 16\% lactofeed (Volac International Ltd., Orwel, Royston, UK; $95.5 \%$ dry matter, $12.5 \%$ protein, $5.0 \%$ oil, $9.0 \%$ ash, $1.0 \%$ fiber, $70 \%$ lactose and a $\mathrm{pH}$ of 6.5-7) for 3 weeks [76]. This result suggests that lactose is not well digested by older pigs due to low lactase activity, and is most probably fermented, which results in a reduced energetic contribution of lactose to the pig. It is hypothesized that excessive intake of lactose may result in a possible overloading of the lactase digestion system which may result in excessive quantities of rapidly fermentable carbohydrate entering the cecum and colon which may exceed the fermentative capacity of the pig [39]. Visscher et al. demonstrated an increased lactic acid concentration in the cecum of $130 \mathrm{~kg}$ pigs fed a diet containing $16.9 \%$ whey powder for 4 weeks and this coincided with a reduction in Lawsonia intracellularis in the cecum. This indicates that lactose was not digested by the fattening pig, rather it was fermented. Furthermore, the increased lactic acid coincided with a reduction in Lawsonia intracellularis, which is a pathogen that can cause intestinal damage and disease in swine [38]. This study, therefore, further demonstrates the potential prebiotic effect from lactose fermentation. Furthermore, a high nutrient digestibility and nitrogen retention by grow-finish pigs fed a diet containing $12 \%$ lactofeed was observed which indicated that this level of lactose is optimal in terms of improving dietary protein utilization and reducing nitrogen excretion, but had no positive effects on ADFI and ADG of pigs [76]. However, there was no further increase in lactobacilli concentrations above $4 \%$ lactofeed in diets, indicating that small quantities of dietary lactose are sufficient to alter conditions in the distal small intestine and large intestine due to low lactase activity in growing-finishing pigs. This also further corroborates our hypothesis that lactase activity is independent of the levels of dietary lactose fed as well as the duration of lactose feeding [5] and that there is a balance between optimal fermentation of lactose and excessive fermentation of lactose that positively, or negatively, effect gastrointestinal health.

\section{Hypothesized modes of action of dietary lactose in weanling pigs}

Based on the comprehensive evidence demonstrated above, the proposed mode of action of dietary lactose in relation to growth performance, gut health, and risk of diarrhea in weanling pigs was demonstrated in this review (Fig. 4). The results of the meta-analysis showed no limitation of dietary lactose concentration on pig growth performance from d 0-7 post-wean. These results, taken together with the other effects of lactose reported in the literature, indicate that dietary lactose stimulates feed

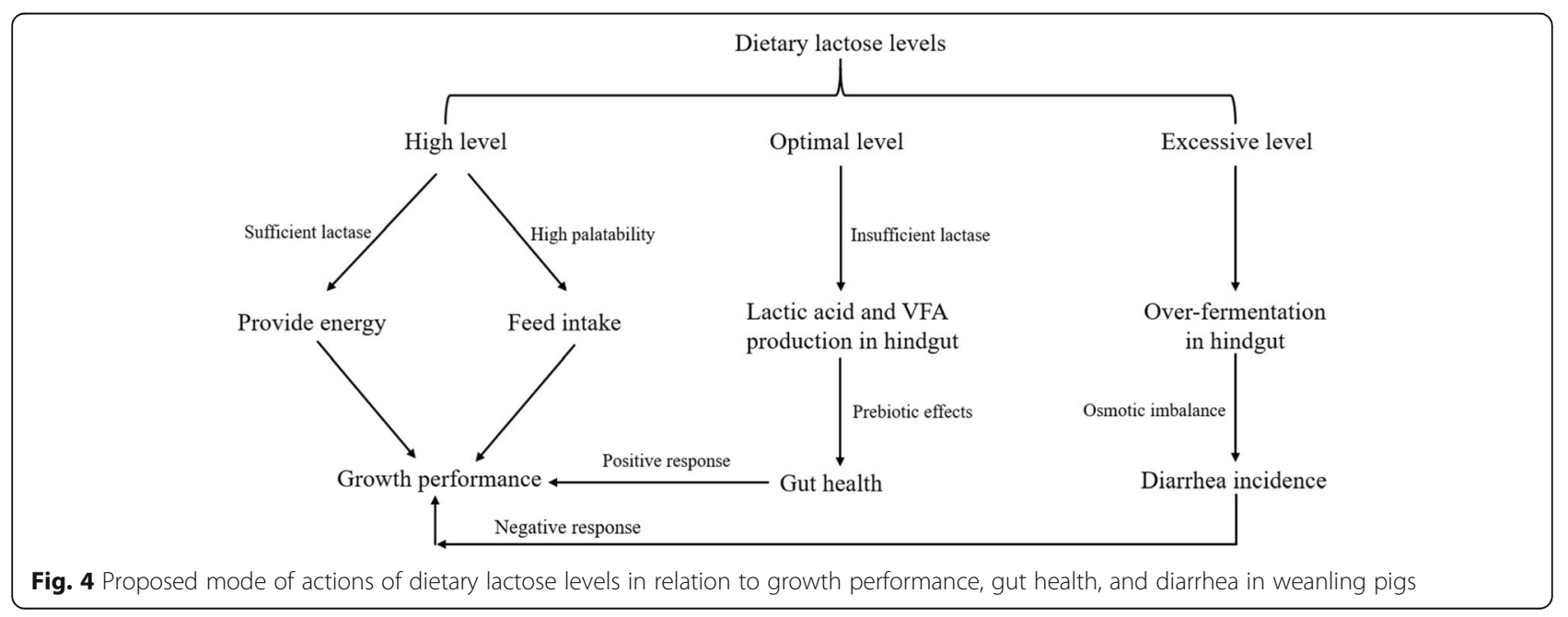


intake due to a high palatability and improves growth performance due to high lactase activity in the early post-wean period.

Secondly, dietary lactose can be fermented by gut microbiota to produce lactic acid and VFA. Therefore, a slightly higher, but optimal level of dietary lactose will provide lactose in quantities greater than lactase can sufficiently digest thereby making lactose a rapidly fermentable carbohydrate source which has the prebiotic effect on the microflora promoting lactic acid and VFA production which stimulates gut health. This hypothesized mode of action is also supported from the meta-analysis because pig ADG was reduced when dietary lactose level exceeded $15 \%$ in diets fed from d 7-14 post-wean, but pig ADFI continued to gradually increase up to $30 \%$ lactose inclusion in the diet. Furthermore, a negative response to dietary lactose on pig ADG and ADFI from $d$ 14-35 post-wean was observed in the models based on the meta-analysis. Taken together, an excessive level of lactose fed from d 14-35 post-wean may induce an osmotic imbalance due to limited lactase activity and excessive lactose fermentation and this may lead to diarrhea. These different mechanisms of action of dietary lactose in weanling pig nutrition are only our hypothesis based on models from the meta-analysis and the normal physiology of pigs, such as a reduced lactase activity and dynamic gut microbiota community as pigs get older. To our knowledge these hypotheses have not been directly tested because it is difficult to quantify lactose in intestinal digesta using current laboratory techniques. No studies have reported how much lactose is digested by endogenous lactase activity and how much lactose is fermented to exert prebiotic effects or lead to diarrhea when weanling pigs are fed different amounts of lactose.

\section{Lactose sources and equivalents in diets}

There are many sources of lactose that are used in weanling pig diets such as pure lactose, dry whey powder (both acid and sweet), whey permeate, deproteinized whey, and milk products such as skimmed milk powder, which show different impacts on growth performance on weanling pigs. A previous study reported pigs weaned at $14 \mathrm{~d}$ of age and BW of $4.4 \mathrm{~kg}$ fed a diet containing $20 \%$ dry whey powder had greater growth performance compared with pigs fed other lactose sources because of the high quality of protein and palatability [60]. However, Delgado et al. [77] indicated pure lactose or whey permeate could replace dry whey powder to maintain the same level of dietary lactose without any negative effect on growth performance of weanling pigs. The inconsistent response observed when using lactose sources which contain protein (i.e. whey powder) may be due to heat damage of the protein and sugar present in the lactose source [78]. The majority of lactose sources used in pig diets go through a drying step in their production [79]. This drying step, along with the high concentration of protein and reducing sugars, make these products highly susceptible to Maillard reactions [78]. On the one hand, previous studies have reported that Maillard reaction products exert prebiotic effects on host health such as antibacterial and antioxidant functions [80, 81]. On the other hand, the Maillard reaction results in a complex that renders the protein, most notably lysine, and sugar unavailable to the animal, thereby decreasing nutrient (lysine and energy) availability, and, thus, results in a loss of performance [82]. The Maillard reaction may explain some inconsistencies in growth performance of pigs fed different concentrations of lactose reported in the literature. Thus, the Maillard reaction should be considered in future studies using lactose sources which contain protein and reducing sugars. The potential of the Maillard reaction was not accounted for in the present meta-analysis because it was not reported in the studies and the majority of the studies fed mash diets where pure lactose was used to increase lactose concentration in the diet. Therefore, pig growth performance responses from different lactose levels was most likely not influenced by the existence of Maillard reaction products in our meta-analysis.

In most nursery pig production systems, it is expensive to provide a diet containing a high level of lactose as recommended in this review. Thus, it is crucial to optimize feed formulations of weanling pig diets by choosing suitable lactose sources and/or lactose equivalents that may partially replace the hypothesized modes of action of lactose. From this review, it was suggested that the modes of action of lactose to improve growth performance of pigs may be partially attributed to its' high palatability to increase feed intake, high digestibility to provide energy, and prebiotic effects to improve gut health. Many different ingredients, additives, and nutrients can be used to replace at least one of the hypothesized modes of action of lactose in weanling pig nutrition. These are so called "lactose equivalents". For example, dietary fiber, resistant starch and non-digestible oligosaccharides exert prebiotic effects on gut microbiota and health of weanling pigs [83]. Some sugars, like dextrose, sucrose, molasses, and extruded starch can be used to increase ADFI and ADG of weanling pigs because they are an easily digestible energy source and also have a high palatability [84, 85]. Previous reports have indicated that supplementation of dietary fiber, dextrose, sucrose, and other low or non-lactose containing milk by-products can be used to partially replace lactose without any negative effect on growth performance of weanling piglets [26, 86]. Mavromichalis et al. [86] showed a combination of sucrose and molasses could effectively replace $50 \%$ and $100 \%$ of 
lactose in diets fed to weanling pigs with average initial BW of $4.6 \mathrm{~kg}$ due to the high palatability and digestibility of sucrose and molasses. Guo et al. [87] demonstrated a candy coproduct from chocolate as an energy source used to substitute up to $45 \%$ of whey permeate had no negative impact on growth performance of weanling pigs. Overall, it is difficult to completely replace dietary lactose due to a high activity of lactase immediately at weaning and its different mechanisms of action in young pigs. In addition, it should be noted that impacts of dietary lactose on subsequent growth performance of pigs is still unknown when pigs have been fed optimal quantities of lactose in the nursery period. Therefore, the potential carry-over effects of lactose and lactose equivalents on performance of growing-finishing pigs should also be considered when the replacement efficiency of lactose equivalents for weanling pigs are studied.

\section{Conclusions}

Dietary lactose plays a crucial role in improving performance and gastrointestinal environment and health of newly weaned pigs. The positive effect of dietary lactose on the growth performance of weanling pigs is most evident in the first 2 weeks after weaning. This coincides with physiological endogenous lactase activity over time, which is independent of dietary lactose level and duration of lactose ingestion. No significant responses, even negative responses, of dietary lactose levels on growth performance and physiological function of pigs after $d$ 14 post-wean may be associated with a reduced lactase activity and excessive fermentation of lactose in pig intestine, resulting in abnormal intestinal permeability. It is recommended that dietary concentrations of lactose should be $20 \%, 15 \%$, and 0 for piglets from d $0-7$, d $7-$ 14 , and d 14-35 post-wean, respectively, based on our meta-analysis. In most situations, the cost of lactose limits its use and commercial nursery pig diets typically do not reach these recommended levels. This is because other ingredients, additives and nutrients can potentially replace lactose. We hypothesized the potential mechanisms of action of lactose fed to weanling pigs to be attributed to the high palatability, high digestibility, and prebiotic effects of lactose to better understand how to use lactose equivalents in the diet. The findings described in this review indicated that dextrose, sucrose, dietary fiber, prebiotics, and milk by-products can partially or completely replace lactose in weanling pig diets. Furthermore, responses to lactose in weanling pigs are influenced by other nutrients, feed additives and AGP supplementation in diets. Therefore, it is also important and necessary to evaluate interactions between lactose and other nutrients or feed additives.

\section{Supplementary Information}

The online version contains supplementary material available at https://doi. org/10.1186/s40104-020-00522-6.

Additional file 1: Supplementary Figure 1. Meta-analysis for the quadratic response of dietary lactose level on average daily feed intake (ADFl, g/d), average daily gain (ADG, g/d) and G:F (ADG:ADFI) of weanling pigs. Meta-analysis was performed using GLM procedure of SAS 9.4. A total of 7 studies containing 54 experimental diets with antibiotic growth promoter (AGP) addition and 4 studies containing 18 experimental diets without AGP were used to develop models to predict the response of lactose level on growth performance of pigs between d 0-14 post-wean. A total of 5 studies containing 28 experimental diets with AGP addition and 5 studies containing 26 experimental diets without AGP were used to develop models to predict the response of lactose level on growth performance of pigs between d 14-35 post-wean.

\section{Abbreviations}

ADFI: Average daily feed intake; ADG: Average daily gain; AGP: Antibiotic growth promoters; ATTD: Apparent total tract digestibility; DM: Dry matter; GE: Gross energy; VFA: Volatile fatty acid

\section{Acknowledgements}

Not applicable.

\section{Authors' contributions}

$\mathrm{NJ}$ and SZ conceived of the manuscript's purpose and design. NJ and GP critically revised the manuscript. JZ collected previous literature and wrote the manuscript. ZZ was responsible for the meta-analysis of publication. All authors read and approved the final manuscript.

\section{Funding}

Not applicable.

\section{Availability of data and materials}

Please contact author for data requests.

Ethics approval and consent to participate

Not applicable.

Consent for publication

Not applicable.

\section{Competing interests}

The authors declare that they have no competing interests.

\section{Author details}

${ }^{1}$ State Key Laboratory of Animal Nutrition, College of Animal Science and Technology, China Agricultural University, No. 2 Yuanmingyuan West Road, Beijing 100193, China. ${ }^{2}$ Trouw Nutrition Innovation, Stationsstraat 77, 3800AG Amersfoort, Netherlands.

Received: 12 May 2020 Accepted: 16 November 2020

Published online: 11 January 2021

\section{References}

1. Vente-Spreeuwenberg MAM, Verdonk JMAJ, Verstegen MWA, Beynen AC. Villus height and gut development in weaned piglets receiving diets containing either glucose, lactose or starch. Brit J Nutr. 2003;90:907-13.

2. Kim YY, Kil DY, Oh HK, Han IK. Acidifier as an alternative material to antibiotics in animal feed. Asian Australas J Anim Sci. 2005;18:1048-60.

3. Suryanarayana MVAN, Suresh J, Rajasekhar MV. Organic acids in swine feeding-a review. Agric Sci Res J. 2012;2:523-33.

4. Pluske JR, Kerton DJ, Cranwell PD, Campbell RG, Mullan BP, King RH, et al. Age, sex, weight at weaning influences organ weight and gastrointestinal development of weanling pigs. Aust J Agric Res. 2003;54:515-27.

5. Forsgård RA. Lactose digestion in human: intestinal lactase appears to be constitutive whereas the colonic microbiome is adaptable. Am J Clin Nutr. 2019;110:273-9. 
6. Zeng X, Sunkara LT, Jiang W, Carter S, Ma X, Qiao S, et al. Induction of porcine host defense peptide gene expression by short-chain fatty acids and their analogs. PLoS One. 2013:8:e72922.

7. Liu H, Wang J, He T, Becker S, Zhang G, Li D, et al. Butyrate: a double-edged sword for health? Adv Nutr. 2018;9:21-9.

8. Szilagyi A. Redefining lactose as a conditional prebiotic. Can J Gastroenterol. 2004;18:163-7.

9. Macfarlane GT, Steed H, Macfarlane S. Bacterial metabolism and healthrelated effects of galacto-oligosaccharides and other prebiotics. J Appl Microbiol. 2008;104:305-44.

10. Venema K. Intestinal fermentation of lactose and prebiotic lactose derivatives, including human milk oligosaccharides. Int Dairy J. 2012;22:123-40.

11. Bindels LB, Delzenne NM, Cani PD, Walter J. Towards a more comprehensive concept for prebiotics. Nat Rev Gastroenterol Hepatol. 2015; 12:303-10.

12. Fukuda S, Toh H, Hase K, Oshima K, Nakanishi Y, Yoshimura K, et al. Bifidobacteria can protect from enteropathogenic infection through production of acetate. Nature. 2011;469:543-7.

13. NRC. Nutrient requirements of swine. 11th ed. Washington: The Nation Academies Press; 2012

14. Jawad R, Elleman C, Vermeer L, Drake A, Woodhead B, Martin G, et al. The measurement of the $\beta / \alpha$ anomer composition within amorphous lactose prepared by spray and freeze drying using a simple $1 \mathrm{H}-\mathrm{NMR}$ method. Pharm Res. 2011;29:511-24.

15. Altamimi MJ, Wolff K, Nokhodchi A, Martin GP, Royall PG. Variability in the a and $\beta$ anomer content of commercially available lactose. Int J Pharm. 2019; 555:237-49.

16. Blackburn DG, Hayssen V, Murphy CJ. The origins of lactation and the evaluation of milk: a review with new hypotheses. Mammal Rev. 1989; 19:1-26.

17. Schaafsma G. Lactose and lactose derivatives as bioactive ingredients in human nutrition. Int Dairy J. 2008;110:1101-11.

18. Moser SA, Tokach MD, Dritz SS, Goodband RD, Nelssen JL, Loughmiller JA. The effects of branched-chain amino acids on sow and litter performance. J Anim Sci. 2000;78:658-67.

19. Park MS, Shinde PL, Yang YX, Kim JS, Chol JY, Yun K, et al. Reproductive performance, milk composition, blood metabolites and hormone profiles of lactating sows fed diets with different cereal and fat sources. Asian-Australas J Anim Sci. 2010;23:226-33.

20. Salcedo J, Frese SA, Mills DA, Barile D. Characterization of porcine milk oligosaccharides during early lactation and their relation to the fecal microbiome. J Dairy Sci. 2016;99:7733-43.

21. Lee S, Joo Y, Lee J, Ha Y, Yeo J, Kim W. Dietary conjugated linoleic acid (CLA) increases milk yield without losing body weight in lactating sows. J Anim Sci Technol. 2014;56:11.

22. Stelwagen K. Mammary gland, milk biosynthesis and secretion: lactose. Encyclopedia Dairy Sci Reference Module Food Sci. 2011. 359-66. https:// doi.org/10.1016/B978-0-12-374407-4.00293-4.

23. Zhao L, Li Y, Li Z, Wu S, Huang K, Chen J, et al. Effect of the valine-to-lysine ratio on the performance of sows and piglets in a hot, humid environment. J Therm Biol. 2019:81:89-97.

24. Dunshea FR, Bauman DE, Nugent EA, Kerton DJ, King RH, McCauley I. Hyperinsulinaemia and branched-chain amino acids when combined can increase milk protein yield in lactating sows. Brit J Nutr. 2005;93:325-32.

25. Dourmad JY, Noblet J, Étienne M. Effect of protein and lysine supple on performance, nitrogen balance, and body composition changes of sows during lactation. J Anim Sci. 1998;76:542-50.

26. Zhang RF, Hu Q, Li PF, Xue LF, Piao XS, Li DF. Effects of lysine intake during middle to late gestation (day 30 to 110) on reproductive performance, colostrum composition, blood metabolites and hormones of multiparous sows. Asian-Australas J Anim Sci. 2011;24:1142-7.

27. Yang YX, Heo S, Jin Z, Yun J, Shinde P, Choi J, et al. Effects of dietary energy and lysine intake during late gestation and lactation on blood metabolites, hormones, milk composition and reproductive performance in multiparous sows. Arch Anim Nutr. 2008;62:10-21.

28. Øverland M, Bikker P, Fledderus J. Potassium diformate in the diets of reproducing sows: effect on performance of sows and litters. Livest Sci. 2009:122:241-7.

29. Ramis G, Evangelista JNB, Quereda JJ, Pallarés FJ, De La Fuente JM, Luna AM. Use of betaine in gilts and sows during lactation: effects on milk quality, reproductive parameters, and piglet performance. J Swine Health Prod. 2011:19:226-32.

30. Montgomery RK, Krasinski SD, Hirschhom JN, Grand RJ. Lactose and lactasewho is lactose intolerant and why? J Pediatr Gastroenterol Nutr. 2007:45: S131-7.

31. Zhang HZ, Malo C, Boyle CR, Buddington RK. Diet influences development of the pig (Sus scrofa) intestine during the first 6 hours after birth. J Nutr. 1998;128:1302-10.

32. De Vos M, Huygelen V, Willemen S, Fransen E, Casteleyn C, Van Cruchten S, et al. Artificial rearing of piglets: effect on small intestinal morphology and digestion capacity. Livest Sci. 2014;159:165-73.

33. Pierce KM, Sweeney T, Brophy PO, Callan JJ, Mccarthy PO, Doherty JV. Dietary manipulation post weaning to improve piglet performance and gastro-intestinal health. Anim Sci. 2005b;81:347-56.

34. Kidder DE, Manners MJ. The level and distribution of carbohydrates in the small intestine mucosa of pigs from weeks of age to maturity. Brit J Nutr. 1980;80:141-53.

35. Elia M, Cummings JH. Physiological aspects of energy metabolism and gastrointestinal effects of carbohydrates. Eur J Clin Nutr. 2007;61:540-74.

36. Montalto M, Curigliano V, Santoro I, Vastola M, Cammarota G, Manna R, et al. Management and treatment of lactose malabsorption. World J Gastroenterol. 2006;12:187-91.

37. Gänzle MG, Follador R. Metabolism of oligosaccharides in lactobacilli: a review. Front Microbiol. 2013;3:340.

38. Visscher C, Kruse A, Sander S, Keller C, Mischok J, Tabeling R, et al. Experimental studies on effects of diet on Lawsonia intracellularis infections in fattening boars in a natural infection model. Acta Vet Scand. 2018;60:22.

39. Williams BA, Verstegen MWA, Tamminga S. Fermentation in the large intestine of single-stomached animal and its relationship to animal health. Nutr Res Rev. 2001;14:207-27.

40. Tolhurst G, Heffron H, Lam YS, Parker HE, Habib AM, Diakogiannaki E, et al. Short-chain fatty acids stimulate glucagon-like peptide-1 secretion via the G-protein-coupled receptor FFAR2. Diabetes. 2012;61:364-71.

41. Gao Z, Yin J, Zhang J, Ward RE, Martin RJ, Lefevre M, et al. Butyrate improves insulin sensitivity and increases energy expenditure in mice. Diabetes. 2009:58:1509-17.

42. Koh A, De Vadder F, Kovatcheva-Datchary P, Bäckhed F. From dietary fiber to host physiology: short-chain fatty acids as key bacterial metabolites. Cell. 2016;165:1332-45

43. Nessmith WB, Nelssen JL, Tokach MD, Goodband RD, Bergstrom JR, Dritz SS, et al. Evaluation of the interrelationships among lactose and protein sources in diets for segregated early-weaned pigs. J Anim Sci. 1997b;75:3214-21.

44. Partridge GG, Gill BP. New approaches with pig weaner diets. In: Wiseman J, Garnsworthy PC, editors. Recent advances in animal nutrition. Nottingham: Nottingham University Press; 1990. p. 221-48.

45. Beasley DE, Koltz AM, Lambert JE, Fierer N, Dunn RR. The evolution of stomach acidity and its relevance to the human microbiome. PLoS One. 2015;10:e0134116.

46. O'Doherty JV, Nolan CS, Callan JJ, Mccarthy P. The interaction between lactofeed level and soya-bean meal on growth performance of weanling pigs. Anim Sci. 2004;78:419-27.

47. O'Doherty JV, Dillon S, Figat S, Callan JJ, Sweeney T. The effects of lactose inclusion and seaweed extract derived from Laminaria spp. on performance, digestibility of diet components and microbial populations in newly weaned pigs. Anim Feed Sci Technol. 2010;157:173-80.

48. Kim JS, Shinde PL, Yang YX, Choi JY, Lohakare JD, Chae BJ. Effects of dietary lactose levels during different starter phases on the performance of weaning pigs. Livest Sci. 2010;131:175-82.

49. Pierce KM, Callan JJ, MaCarthy P, O'Doherty JV. Performance of weanling pigs offered low and high lactose diets supplemented with avilamycin or inulin. Anim Sci. 2005a;80:313-8.

50. Mahan DC, Newton EA. Evaluation of feed grains with dried skim milk and added carbohydrate source on weanling pig performance. J Anim Sci. 1993;71:3376-82.

51. Bertol TM, Filho Jl, Ludke JV. Dietary lactose levels for weanling pigs. Rev Bras Zootec. 2000;29:1387-97.

52. Mahan DC, Fastinger ND, Peter JC. Effect of diet complexity and dietary lactose levels during three starter phases on post-weaning pig performance. J Anim Sci. 2004:82:2790-7.

53. Yang $X$, Kim JS, Baidoo SK. Dietary effect of lactose on growth performance of nursery pigs on a liquid feeding system. J Anim Sci. 2016;94(supple2):131-2. 
54. Tran H, Moreno R, Hinkle EE, Bundy JW, Walter J, Burkey TE, Miller PS. Effects of lactose and yeast-dried milk on growth performance, fecal microbiota, and immune parameters of nursery pigs. J Anim Sci. 2012;90:3049-59.

55. Toplis P, Wellock IJ, Stewart A, Bailey M, Salmon L. High lactose pellets fed from $4 \mathrm{~d}$ of convey lifetime benefits when compared to a standard preweaning diet. J Anim Sci. 2016;94:18.

56. Pollmann DS, Danielson DM, Peo ER Jr. Effect of Lactobacillus spp. acidophilus on starter pigs fed a diet supplemented with lactose. J Anim Sci. 1980;51:638-44.

57. Jeong YD, Ko HS, Hosseindoust A, Choi YH, Chae BJ, Yu DJ, et al. Effects of dietary lactose levels and supplementation of prebiotics on growth performance in weanling pigs. J Anim Sci. 2018;96(Supple2):42-3.

58. Molino JP, Donzele JL, Miranda RF, De Oliveira RFM, Ferreira AS, De Morae CA, et al. Lactose levels in diets for piglets weaned at 21 days of age. Rev Bras Zootec. 2011:40:1233-41.

59. Cromwell GL, Allee GL, Mahan DC. Assessment of lactose level in the mid- to late-nursery phase on performance of weanling pigs. J Anim Sci. 2008:86:127-33.

60. Nessmith WB, Tokach MD, Goodband RD, Nelssen JL. Defining quality of lactose sources used in swine diets. J Swine Health Prod. 1997a;5:145-9.

61. Pierce KM, Callan JJ, McCarthy P, Doherty JV. The interaction between lactose level and crude protein concentration on piglet post-weaning performance, nitrogen catabolism and faecal volatile fatty acid concentration. Anim Feed Sci Technol. 2007:132:267-82.

62. O'Connell JM, Callan JJ, O'Doherty JV. The interaction between cereal type and lactose level on piglet performance and diet digestibility post weaning. Anim Sci. 2005;81:265-9.

63. St-Pierre NR. Invited review: integrating quantitative findings from multiple studies using mixed model methodology. J Dairy Sci. 2001;84:741-55.

64. Graham A, Greiner L, Knopf B, Goncalves MAD, Cast W, Orlando UAD. Evaluation of the lactose level and organic acid inclusion in newly weaned pigs. J Anim Sci. 2018;96(Supple2):154

65. Lynch MB, Callan JJ, O'Doherty JV. The interaction between dietary crude protein and fermentable carbohydrate source on piglet post weanling performance, diet digestibility and selected faecal microbial populations and volatile fatty acid concentration. Livest Sci. 2009;124:93-100

66. Lynch MB, Callan JJ, O' Doherty JV. The interaction between enzyme supplementation and lactose level on the performance of weanling pigs fed barley based diets. Livest Sci. 2007:108:258-61.

67. Mahan DC. Efficacy of dried whey and its lactalbumin and lactose components at two dietary lysine levels on post weaning pig performance and nitrogen balance. J Anim Sci. 1992;70:2182-7.

68. Bach Knudsen KE. Lactose in diet influences the degradation of mixed linked $\beta$ (1-3; 1-4)-D-glucan in the small intestine of pigs. J Anim Sci. 2012; 90:125-7.

69. Pierce KM, Sweeney T, Brophy PO, Callan JJ, Fitzpatrick E, McCarthy P, et al. The effects of lactose and inulin on intestinal morphology, selected microbial populations and volatile fatty acid concentrations in the gastrointestinal tract of the weanling pig. Anim Sci. 2006b;82:311-8.

70. Acosta JA, Gabler NK, Frank JW, Bass BE, Patience JE. Effect of lactose, inulin, lactobacillus acidophilus fermentation product, or dietary antibiotics on intestinal function of nursery pigs. J Anim Sci. 2017:95(Supple 2):149.

71. Spreeuwenberg MAM, Verdonk JMAJ, Gaskins HR, Verstegen MWA. Small intestine epithelial barrier function is compromised in pigs with low feed intake at weaning. J Nutr. 2001;131:1520-7.

72. Charalampopoulos D, Wang R, Pandiella SS, Webb C. Application of cereals and cereal components in functional foods: a review. Int J Food Microbiol. 2002:79:131-41

73. Zhao JB, Bai Y, Tao SY, Zhang G, Liu L, Zhang S. Fiber-rich foods affects gut bacterial community and short-chain fatty acids production in pig model. J Funct Foods. 2019;57:266-74.

74. Konstantinov SR, Awati A, Smidt H, Williams BA, Akkermans ADL, De Vos WM. Specific response of a novel and abundant Lactobacillus amylovoruslike phylotype to dietary prebiotics in the guts of weaning piglets. Appl Environ Microbiol. 2004:70:3821-30

75. Jiang T, Mustapha A, Savaiano DA. Improvement of lactose digestion in humans by ingestion of unfermented milk containing Bifidobacterium longum. J Dairy Sci. 1996;79:750-7.

76. Pierce KM, Sweeney T, Callan JJ, Byrne C, McCarthy P, O'Doherty JV. The effect of inclusion of a high lactose supplement in finishing diets on nutrient digestibility, nitrogen excretion, volatile fatty acid concentrations and ammonia emission from boars. Anim Feed Sci Technol. 2006a;125:45-60.
77. Delgado L, Oña C, Naranjo A, Quisirumbay J, Aragón E. Productive performance of piglets fed with different sources of lactose. J Agr Life Sci. 2016;3:51-4

78. Van Bekel MAJS. Effect of heating on Maillard reactions in milk. Food Chem. 1998:62:403-14

79. Barba D, Beolchini F, Cifoni D, Veglió F. Whey protein concentrate production in a pilot scale two-stage diafiltration process. Sep Sci Technol. 2001;36:587-603.

80. Carmelina S, Josephine M, Bettina C, Annette F, Martina P, Szillard K, et al. Radical scavenging activity, anti-bacterial and mutagenic effects of cocoa bean Maillard reaction products with degree of roasting. Mol Nutr Food Res. 2008;52:342-51.

81. Somoza V. Five years of research on health risks and benefits of Maillard reaction products: an update. Mol Nutr Food Res. 2005;49:663-72.

82. Hulshof TG, Bikker $P$, van der Poel AFB, Dendriks WH. Assessment of protein quality of soybean meal and 00-rapeseed meal toasted in the presence of lignosulfonate by amino acid digestibility in growing pigs and Maillard reaction products. J Anim Sci. 2016;94:1020-30.

83. Zhao JB, Liu P, Wu Y, Guo PT, Liu L, Ma N, et al. Dietary fiber increases butyrate-producing bacteria and improves the growth performance of weaned piglets. J Agric Food Chem. 2018;66:7995-8004.

84. Guzman-Pino SA, Sola-Oriol D, Figueroa J, Dwyer DM, Perez JF. Effect of a long-term exposure to concentrated sucrose and maltodextrin solutions on the preference appetence, feed intake and growth performance of postweaned piglets. Physiol Behav. 2015;141:85-91.

85. Roura E, Fu M. Taste, nutrient sensing and feed intake in pigs (130 years of research: then, now and future). Anim Feed Sci Technol. 2017;233:3-12.

86. Mavromichalis I, Hancock JD, Hines RH, Senen BW, Cao H. Lactose, sucrose, and molasses in simple and complex diets for nursery pigs. Anim Feed Sci Technol. 2001;93:127-35.

87. Guo JY, Phillips CE, Coffey MT, Kim SW. Efficacy of a supplemental candy coproduct as an alternative carbohydrate source to lactose on growth performance of newly weaned pigs in a commercial farm condition. J Anim Sci. 2015;93:5304-12
Ready to submit your research? Choose BMC and benefit from:

- fast, convenient online submission

- thorough peer review by experienced researchers in your field

- rapid publication on acceptance

- support for research data, including large and complex data types

- gold Open Access which fosters wider collaboration and increased citations

- maximum visibility for your research: over $100 \mathrm{M}$ website views per year

At $\mathrm{BMC}$, research is always in progress.

Learn more biomedcentral.com/submissions 